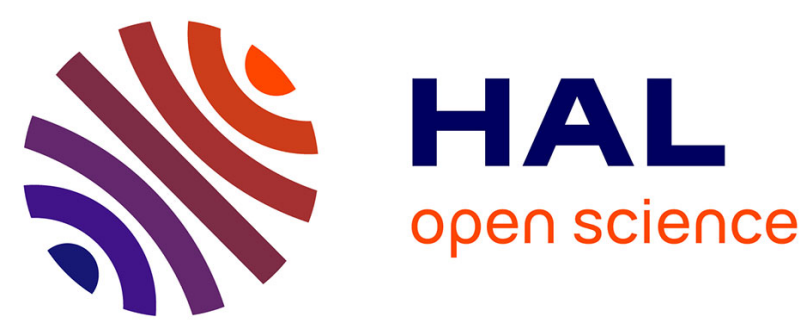

\title{
Transient heat conduction within periodic heterogeneous media: A space-time homogenization approach
}

Abdelghani Matine, Nicolas Boyard, Grégory Legrain, Yvon Jarny, Patrice Cartraud

\section{- To cite this version:}

Abdelghani Matine, Nicolas Boyard, Grégory Legrain, Yvon Jarny, Patrice Cartraud. Transient heat conduction within periodic heterogeneous media: A space-time homogenization approach. International Journal of Thermal Sciences, 2015, 92, pp.217-229. 10.1016/j.ijthermalsci.2015.01.026 . hal01317644

\section{HAL Id: hal-01317644 \\ https://hal.science/hal-01317644}

Submitted on 15 Jul 2021

HAL is a multi-disciplinary open access archive for the deposit and dissemination of scientific research documents, whether they are published or not. The documents may come from teaching and research institutions in France or abroad, or from public or private research centers.
L'archive ouverte pluridisciplinaire HAL, est destinée au dépôt et à la diffusion de documents scientifiques de niveau recherche, publiés ou non, émanant des établissements d'enseignement et de recherche français ou étrangers, des laboratoires publics ou privés. 


\title{
Transient heat conduction within periodic heterogeneous media A space-time homogenization approach
}

\author{
A. MATINE ${ }^{1}$,N. BOYARD ${ }^{1,}{ }^{*}$, G. LEGRAIN ${ }^{2}$, Y. JARNY ${ }^{1}$, P. CARTRAUD ${ }^{2}$
}

${ }^{1}$ Université de Nantes, Nantes Atlantique Universités, Laboratoire de Thermocinétique de Nantes, UMR CNRS 6607, La Chantrerie, rue Christian Pauc, BP 50609, 44306 Nantes cedex

3, France.

${ }^{2}$ Institut de Recherche en Génie civil et Mécanique (GeM), UMR CNRS 6183 Ecole Centrale de Nantes, BP 92101, 44321 Nantes cedex 3, France

*Corresponding author.e-mail: yvon.jarny@univ-nantes.fr

Phone: 0033 (0)2 406831 11, Fax: 0033 (0)2 40683141

\begin{abstract}
Composite materials generally exhibit a highly anisotropic thermal behavior (due to the orientation of fibers), leading to strong difficulties to determine the thermal conductivity tensor. Two approaches can be developed for its evaluation. The first one is to carry out experimental measurements with one or several devices to get all the components of the tensor. The second one is to use predictive models based on homogenization theories from the properties and the arrangement at the microscopic scale of each component of the composite material.

Following this second approach, a space-time homogenization approach, based on the multiscale asymptotic expansion method, is first developed to model the transient heat conduction problem within periodic heterogeneous structures. The introduction of additional terms to correct the edge effects (i.e. close to the boundaries of the macroscopic domain) in transient state is considered. We show how these transient correcting terms can be introduced and calculated, depending on the classical boundary conditions in conduction heat transfer problems. Moreover, we underline that correcting terms have also to be added to take into account "short time" effects.

Furthermore, we propose to discuss numerical results of the heat transfer modeling in a Laser Flash experiment. We specifically show how the effective thermal diffusivity may be biased when edge effects are neglected in the homogenized model.
\end{abstract}

Keywords: heat conduction, composite structures, homogenization theory, multi-scale model, short-time effects, spatial edge effects 


\section{1.-Introduction}

In order to predict the thermal (and the associated thermo-mechanical) behavior within complex heterogeneous media, a usual approach consists in determining the effective thermal properties and/or temperature fields through homogenized heat transfer models. This approach is very interesting in several industrial domains such as in aeronautics or automotive. A typical example is the design of complex parts for new airplane structure where the use of composite materials with highly anisotropic thermal properties (due to the orientation of fibers) becomes more and more systematic [1]. However, the determination of the effective thermal conductivity tensor is a tricky task. Consequently, reliable and efficient methods, initially developed for mechanical models [2], are thus required for its determination. From an experimental point of view, specific devices such as classical transient laser flash (LF) [3]- [4], hot wire [5] methods, or a specific hot disc method [6] can be used to estimate the effective thermal properties. Another possibility is to make calculations from a representative volume element [7] of the anisotropic actual medium, knowing the thermal conductivity of each phase.

The heat transfer modeling according to a multi-scale analysis [8]-[9]-[10] is complementary to the experimental approach and quite powerful. It aims on one hand to determine the effective thermal properties from data known at the scale of the components, and on the other hand to have a better understanding of the "edge effects" [11]-[14] which may disrupt the temperature field of the homogenized heat conduction model close to the boundaries of the spatial domain. To our knowledge, this second aspect is rarely discussed in the literature. Consequently, within the framework of the estimation process of effective properties, which would result on the comparison of experimental surface temperatures with the solutions of homogenized models, more insight have to be done in the analysis of these "edge effects", to know when they can be neglected or not. More generally, it is well-known that errors associated to modeling in the inverse analysis of experimental data should imply biased estimation (systematical errors) of the unknown model parameters [15].

A previous work [13], devoted to the heat conduction steady state analysis within heterogeneous periodic structures, shows how correcting terms can be introduced in the multiscale asymptotic method to take into account these "edge effects", in the case of a homogenized 3-D heat conduction problem. The results were obtained by following the works of Dumontet [14] in elasticity.

In this paper, a space-time homogenization approach, based on the multi-scale asymptotic expansion method, is developed first to model the transient heat conduction problem within periodic structures. Such approach was also studied in [16-17]. However, the introduction of additional terms to correct the edge effects in transient state was not considered. We show how these transient correcting terms can be introduced and calculated, depending on the classical boundary conditions in heat transfer problems. Moreover, correcting terms have also to be added to take into account "short time" effects. Numerical results are presented in the case of a simple multilayered media, but the method is quite general and it could be used for periodic heterogeneous structures, like in plain weave fabric composites [18].

The last section is devoted to the discussion of numerical results of the heat transfer modeling in a LF experiment where numerical data of Fudym et al [11] are thus used for this purpose. The heterogeneous solution is compared to the homogenized one, computed with and without correcting terms, and to the analytical homogeneous solution. Specifically, the LF method is based on the heating of the front surface of a thin sample (with parallel faces) with a nearly instantaneous pulse of light (compared to the heat conduction characteristic time of the medium). The influence of the heat losses by convection is also considered. The temperature rise on the back face is measured as a function of time (thermocouple or IR detector), and it is used to determine the thermal diffusivity (in the direction normal to the back face) of the 
sample. The bias, due to the estimated value of the thermal diffusivity when edge effects are neglected in the homogenized model, is evaluated.

\section{2.- Problem statement -Heat conduction in the heterogeneous material}

Let us consider a piece of heterogeneous periodic material, figure 1 , defined in a bounded domain $\Omega \subset \mathrm{R}^{3}$. The macroscopic coordinates of a point of $\Omega$ are denoted $\boldsymbol{x}=\left(x_{1}, x_{2}, x_{3}\right)$ in a Cartesian coordinate system $\left\{\mathbf{0}, \boldsymbol{e}_{1}, \boldsymbol{e}_{2}, \boldsymbol{e}_{3}\right\}$. The boundary $\partial \Omega$ is subdivided in four distinct parts $\partial \Omega=\cup_{i=1}^{4} \Gamma_{i}$, in order to consider the different usual kinds of boundary conditions associated to the heat conduction problem:

- A Fourier condition on $\Gamma_{1}$ : the normal outward component $\phi^{\varepsilon} . n$ of the heat flux is fixed by an external temperature $T_{\text {ext }}$ and a heat transfer coefficient $h$.

- A Neumann condition on $\Gamma_{2}$ : the normal outward component $\phi^{\varepsilon} . n$ of the heat flux is fixed.

- A Dirichlet condition on $\Gamma_{3}$ : the temperature is imposed.

- A periodic condition on $\Gamma_{4}$.

The initial condition is defined by the field $T_{i n i}(\boldsymbol{x}), \boldsymbol{x} \in \Omega$, which is isothermal or not. A spatially distributed volume heat source $f(\boldsymbol{x}), \boldsymbol{x} \in \Omega$ may be considered all over the spatial domain, at the macroscopic scale.

The heterogeneous fields in the spatial domain $\Omega$, are denoted respectively $T^{\epsilon}$ (temperature) and $\phi^{\varepsilon}$ (heat flux density). These fields over the time interval $\left(0, t_{f}\right)$ satisfy the following set of transient heat conduction equations together with the different kinds of boundary and initial conditions:

$\left\{\begin{array}{c}\rho C_{p} \frac{\partial T^{\varepsilon}(\boldsymbol{x}, t)}{\partial t}-\operatorname{div}_{x}\left[\boldsymbol{\phi}^{\varepsilon}(x, t)\right]=f(x) \quad \text { in } \Omega \times\left(0, t_{f}\right) \\ \phi^{\varepsilon}(\boldsymbol{x}, t)=\boldsymbol{K} \boldsymbol{\nabla}_{\boldsymbol{x}} T^{\varepsilon}(\boldsymbol{x}, t) \quad \text { in } \Omega \times\left(0, t_{f}\right) \\ T^{\varepsilon}(x, t=0)=T_{\text {ini }}(\boldsymbol{x}) \quad \text { in } \Omega \\ \boldsymbol{\phi}^{\varepsilon}(\boldsymbol{x}, t) \cdot n=h\left(T^{\varepsilon}-T_{\text {ext }}\right) \text { on } \Gamma_{1} \times\left(0, t_{f}\right) \\ \boldsymbol{\phi}^{\varepsilon}(\boldsymbol{x}, t) \cdot \boldsymbol{n}=F \quad \text { on } \quad \Gamma_{2} \times\left(0, t_{f}\right) \\ T^{\varepsilon}(x, t)=T_{\text {ext }} \quad \text { on } \Gamma_{3} \times\left(0, t_{f}\right) \\ \text { Periodic conditions on } \Gamma_{4} \times\left(0, t_{f}\right)\end{array}\right.$

where $\boldsymbol{n}$ is the outward normal unit; $\rho, C_{p}$ and $\boldsymbol{K}$ are respectively the density, the heat capacity and the thermal conductivity tensor of the heterogeneous medium which is assumed to have a periodic structure.

The periodic cell (see the figure 1), is denoted $Y=\prod_{i=1}^{3}\left[0, l_{i}\right]$ and $\boldsymbol{y}=\left(y_{1}, y_{2}, y_{3}\right) \in Y$ are the coordinates of a cell point. The scale factor $\varepsilon$ is the ratio between the size of $Y$ and the size of $\Omega \square \square$ the microscopic coordinates are thus defined from $\boldsymbol{y}=\varepsilon^{-1} \boldsymbol{x}$.

Each component $k_{i j}(\boldsymbol{y}) ; i, j=1,2,3$ of the thermal tensor and the parameter $\rho C_{p}(\boldsymbol{y})$ are cellperiodic and depend on the local variable $\boldsymbol{y}$ (microscopic scale) in the cell domain $Y$. 


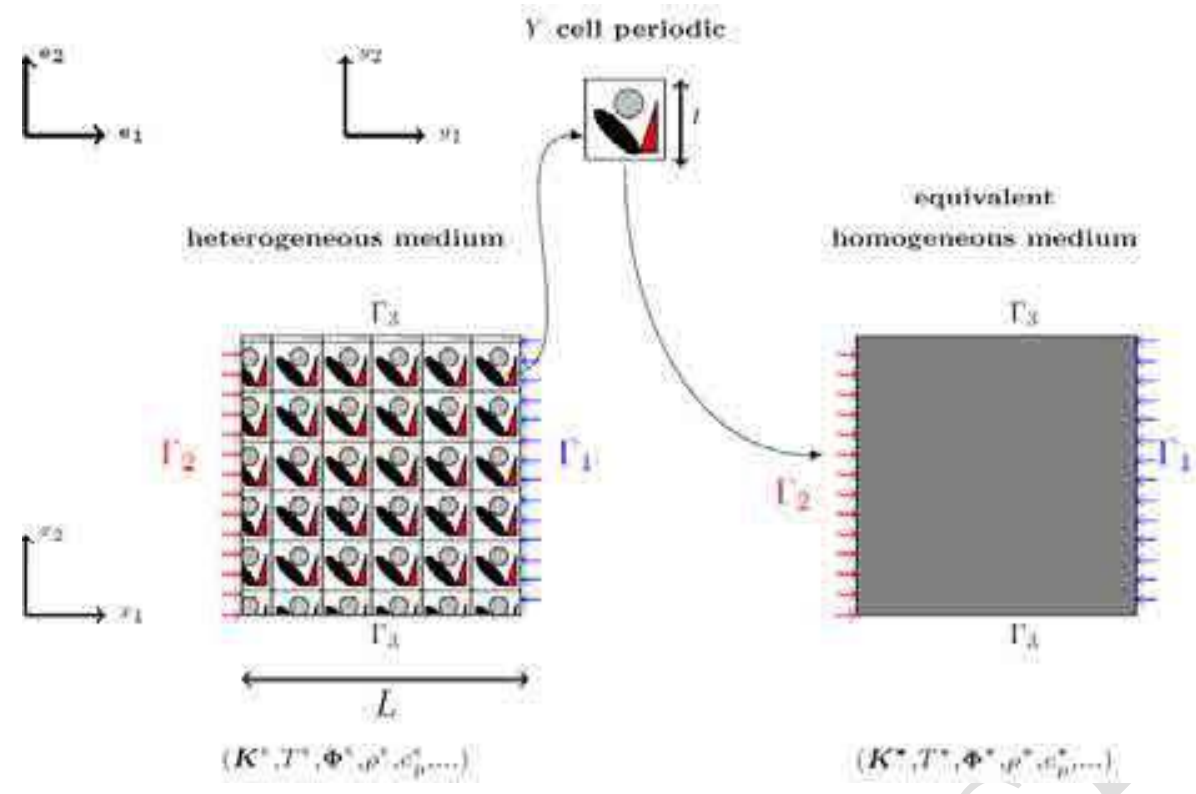

Figure 1: Spatial domain $\Omega$ of the heterogeneous periodic medium and the associated periodic cell $Y$

\section{3.- Periodic Homogenization in Transient State}

\section{1-Multi-scale asymptotic expansion method}

It is assumed that the thermal conductivity of each components of the heterogeneous structure have the same order of magnitude, which means that the thermal contrast is not too large. The same assumption is done for the heat capacities. The influence of large contrast is not considered here and should lead to more developments, as described for example in [10].

Assuming that the scale factor $\varepsilon$ is small enough, the asymptotic expansion method [9] may be used and the temperature $T^{\varepsilon}$ is expanded, like for steady state solutions [13], under the following form:

$$
T^{\varepsilon}(\boldsymbol{x}, \boldsymbol{y}, t)=T^{0}(\boldsymbol{x}, \boldsymbol{y}, t)+\varepsilon T^{1}(\boldsymbol{x}, \boldsymbol{y}, t)+\varepsilon^{2} T^{2}(\boldsymbol{x}, \boldsymbol{y}, t)+\cdots ; \quad \boldsymbol{x} \in \Omega, \boldsymbol{y} \in Y
$$

where $T^{k}$ is the approximation of $T^{\varepsilon}$ at the order $k$ and is supposed to be spatially periodic at the microscopic scale. The time variable appears in the asymptotic development as a simple parameter. Consequently, the heat flux density $\boldsymbol{\phi}^{\varepsilon}(\boldsymbol{x}, t)=\boldsymbol{K} \boldsymbol{\nabla}_{\boldsymbol{x}} T^{\varepsilon}(\boldsymbol{x}, t)$ can be expanded such that:

$\phi^{\varepsilon}(x, y, t)=\varepsilon^{-1} \phi^{-1}(x, y, t)+\phi^{0}(x, y, t)+\varepsilon \phi^{1}(x, y, t)+\cdots$

By injecting the development (2) in the transient heat conduction equation (1), and using the property that an entire series is equal to zero if and only if each of its term is null, [8-10] it results in a new equation for each power of $\varepsilon$ :

- At the order $k=-2$, it comes: $\operatorname{div}_{y}\left[\boldsymbol{K}(\boldsymbol{y}) \boldsymbol{\nabla}_{\boldsymbol{y}} T^{0}(\boldsymbol{x}, \boldsymbol{y}, t)\right]=0$. Furthermore, since $T^{0}$ is periodic, it implies that $\boldsymbol{K}(\boldsymbol{y}) \boldsymbol{\nabla}_{\boldsymbol{y}} T^{0}(\boldsymbol{x}, \boldsymbol{y}, t)=0$. Hence the function $T^{0}$ does not depend on the microscopic variable: $T^{0}(x, y, t)=T^{0}(x, t)$. Consequently, $\boldsymbol{\phi}^{-1}(\boldsymbol{x}, \boldsymbol{y}, t)=\boldsymbol{K}(\boldsymbol{y}) \boldsymbol{\nabla}_{\boldsymbol{y}} T^{0}(\boldsymbol{x}, t)=\mathbf{0}$.

- At the order $k=-1$, it comes: $\operatorname{div}_{y}\left[\boldsymbol{K}(\boldsymbol{y})\left(\boldsymbol{\nabla}_{\boldsymbol{x}} T^{0}(\boldsymbol{x}, t)+\boldsymbol{\nabla}_{\boldsymbol{y}} T^{1}(\boldsymbol{x}, \boldsymbol{y}, t)\right)\right]=0$; the equation satisfied by the function $T^{1}$ is: 


$$
\left\{\begin{array}{c}
\operatorname{div}_{y}\left[\boldsymbol{K}(\boldsymbol{y})\left(\boldsymbol{\nabla}_{\boldsymbol{x}} T^{0}(x, t)+\boldsymbol{\nabla}_{\boldsymbol{y}} T^{1}(\boldsymbol{x}, \boldsymbol{y}, t)\right)\right]=0 \text { in } Y \\
T^{1} \text { periodic on } \partial Y
\end{array}\right.
$$

Consequently, the new variables $\omega_{i}(\boldsymbol{y}), i=1,2,3$ may be introduced at the microscopic scale, such that:

$$
T^{1}(\boldsymbol{x}, \boldsymbol{y}, t)=\sum_{i=1}^{3} \frac{\partial T^{0}(\boldsymbol{x}, t)}{\partial x_{i}} \omega_{i}(\boldsymbol{y})
$$

And they are solution of the cell problems $(i=1,2,3)$ :

$$
\left\{\begin{array}{c}
\operatorname{div}_{y}\left[\boldsymbol{K}(\boldsymbol{y})\left(\boldsymbol{e}_{\boldsymbol{i}}+\boldsymbol{\nabla}_{\boldsymbol{y}} \omega_{i}(\boldsymbol{y})\right)\right]=0 \text { in } Y \\
\omega_{i} \text { periodic on } \partial Y
\end{array}\right.
$$

At the order $\mathrm{k}=0$, we get:

$$
\begin{aligned}
\operatorname{div}_{y}\left[\boldsymbol{K}(\boldsymbol{y})\left(\boldsymbol{\nabla}_{\boldsymbol{x}} T^{1}(\boldsymbol{x}, \boldsymbol{y}, t)+\boldsymbol{\nabla}_{\boldsymbol{y}} T^{2}(\boldsymbol{x}, \boldsymbol{y}, t)\right)\right] \\
=\rho C_{p} \frac{\partial T^{0}(\boldsymbol{x}, t)}{\partial t}-\operatorname{div}_{x}\left[\boldsymbol{K}(\boldsymbol{y})\left(\boldsymbol{\nabla}_{\boldsymbol{y}} T^{1}(\boldsymbol{x}, \boldsymbol{y}, t)+\boldsymbol{\nabla}_{\boldsymbol{x}} T^{0}(\boldsymbol{x}, t)\right)\right] \\
-f(\boldsymbol{x})
\end{aligned}
$$

The function $T^{2}$ exists and is cell periodic if and only if the integral of the left term on the cell $Y$ is equal to zero [8], which leads to:

$$
\begin{array}{r}
\frac{1}{|\boldsymbol{Y}|} \int_{Y} \rho C_{p} \frac{\partial T^{0}(x, t)}{\partial t} d y-\operatorname{div}_{x}\left\{\frac { 1 } { | \boldsymbol { Y } | } \int _ { Y } \left[\boldsymbol { K } ( \boldsymbol { y } ) \sum _ { i = 1 } ^ { 3 } \left(\boldsymbol{e}_{\boldsymbol{i}}\right.\right.\right. \\
\left.\left.\left.+\boldsymbol{\nabla}_{\boldsymbol{y}} \omega_{i}(\boldsymbol{y})\right) \otimes \boldsymbol{e}_{\boldsymbol{i}}\right] d \boldsymbol{y} \boldsymbol{\nabla}_{\boldsymbol{x}} T^{0}(\boldsymbol{x}, t)\right\}=f(x)
\end{array}
$$

Remark: $(\boldsymbol{u} \otimes \boldsymbol{v})$ denotes the dyadic product of two vectors, that is the matrix product of $\boldsymbol{u}$ by $v$, considered respectively as a column matrix and a row matrix.

\section{2- Effective thermal properties}

Finally, by introducing the effective thermal properties of the homogenized medium:

$$
\begin{aligned}
& \left(\rho C_{p}\right)^{*}=\frac{1}{|Y|} \int_{Y} \rho C_{p} d y \\
& \text { and } \boldsymbol{K}^{*}=\frac{1}{|Y|} \int_{Y}\left[\boldsymbol{K}(\boldsymbol{y}) \sum_{i=1}^{3}\left(\boldsymbol{e}_{\boldsymbol{i}}+\boldsymbol{\nabla}_{\boldsymbol{y}} \omega_{i}(\boldsymbol{y})\right) \otimes \boldsymbol{e}_{\boldsymbol{i}}\right] d \boldsymbol{y}
\end{aligned}
$$

The function $T^{0}(x, t)$ satisfies the macroscopic scale heat conduction equation (8), which can be rewritten

$$
\left\{\begin{array}{c}
\left(\rho C_{p}\right)^{*} \frac{\partial T^{0}(\boldsymbol{x}, t)}{\partial t}-\operatorname{div}_{x}\left[\boldsymbol{K}^{*} \nabla_{\boldsymbol{x}} T^{0}(\boldsymbol{x}, t)\right]=f(x) \quad \text { in } \Omega \times\left(0, t_{f}\right) \\
T^{0}(\boldsymbol{x}, t=0)=T_{\text {ini }}(\boldsymbol{x}) \quad \text { in } \Omega
\end{array}\right.
$$


And the heat flux, at the order $k=0$, is given by:

$$
\boldsymbol{\phi}^{\mathbf{0}}(\boldsymbol{x}, \boldsymbol{y}, t)=\left[\boldsymbol{K}(\boldsymbol{y}) \sum_{i=1}^{3}\left(\boldsymbol{e}_{\boldsymbol{i}}+\boldsymbol{\nabla}_{\boldsymbol{y}} \omega_{i}(\boldsymbol{y})\right) \otimes \boldsymbol{e}_{\boldsymbol{i}}\right] \boldsymbol{\nabla}_{\boldsymbol{x}} T^{0}(\boldsymbol{x}, t)
$$

Effective properties (9) are defined with spatial periodic boundary conditions on the cell $Y$, then if no boundary conditions are fixed on $\Gamma \times\left(0, t_{f}\right)$ such periodic conditions would be "naturally" satisfied. When the actual boundary conditions (see equations (1)) are not periodic, "edge effects" appears and specific mathematical developments are required, as demonstrated below.

\section{3- Numerical illustration (Case \#1) of "short time effects"}

The motivation of this first numerical example is to highlight only the "short time effects" which appears in transient state according to this homogenized approach. To illustrate this phenomenon, we consider a very simple bi-layered periodic structure, figure 2, already studied in steady state [13] and we compare the heterogeneous field $T^{\varepsilon}$ with the homogenized solution $\left(T^{0}+\varepsilon T^{1}\right)$. The thermal and geometrical data are given in table 1 . The spatial domain $\Omega$ is a square. To avoid edge effects, the functions $T^{\varepsilon}, T^{0}$ and $T^{1}$ are computed with spatial periodic boundary conditions on $\Gamma \times\left(0, t_{f}\right)$. Without volume heat source $(f \equiv 0)$, we only consider the thermal relaxation phenomena, from a non isothermal initial condition:

$$
T_{\text {ini }}(x)=100 . \Psi_{D}(\boldsymbol{x}), x \in \Omega
$$

where $\Psi_{D}(\boldsymbol{x})=1$, if $x \in D$; $=0$ else; is the spatial characteristic function of the sub-domain $D$, and $D$ is the strip defined by two layers, between $x_{2}=4.5 \mathrm{~mm}$ and $x_{2}=5.5 \mathrm{~mm}$, as shown on figure 2 .

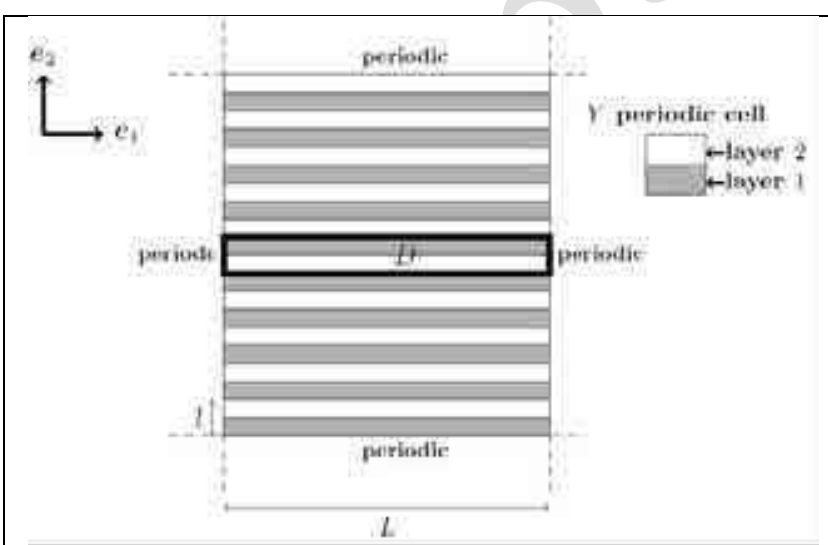

Figure 2: Schematic description of the multilayered medium and the periodic cell- Insulating layer (grey), conductive layer (blank). Case \#1: non isothermal initial condition and periodic boundary conditions.

\begin{tabular}{|l|l|l|}
\hline Layer & Properties & Value \\
\hline \hline \multirow{3}{*}{ Layer 1 } & $k_{m}\left(W \mathrm{~m}^{-1} \mathrm{~K}^{-1}\right)$ & 0.2 \\
& $\rho_{m} \times C p_{m}\left(J \mathrm{~m}^{-3} \mathrm{~K}^{-1}\right)$ & $1365.10^{3}$ \\
$\mathrm{f}$ & 0 \\
\hline \multirow{3}{*}{ Layer 2 } & $k_{f}\left(W \mathrm{~m}^{-1} \mathrm{~K}^{-1}\right)$ & 5 \\
& $\rho_{f} \times C p_{f}\left(J \mathrm{~m}^{-3} \mathrm{~K}^{-1}\right)$ & 1350 \\
& $\mathrm{f}$ & 0 \\
\hline \multirow{5}{*}{ Geometry } & $L(\mathrm{~m})$ & $10^{-2}$ \\
& $I(\mathrm{~m})$ & $10^{-3}$ \\
& $\varepsilon$ & 0.1 \\
\hline
\end{tabular}

Table 1: Thermal and geometrical data for the multilayered periodic structure- Cases \#1 to \#3 $\kappa=k_{f} / k_{m}=25$. 
The multilayered stack is characterized by the thickness $l / 2$ of each layer, by the thermal contrast $=k_{f} / k_{m}$, the ratio of the heat conductivity of the conductive layer over the insulating one, and the ratio of the heat capacities

$$
\begin{gathered}
\boldsymbol{K}(\boldsymbol{y})=\left[\begin{array}{cc}
k_{11} & 0 \\
0 & k_{22}
\end{array}\right], \text { with } k_{i i}(\boldsymbol{y})=\left[\begin{array}{c}
k_{m}, \text { if } \mathrm{y} \in(\text { layer } 1) \\
k_{f}, \text { if } \mathrm{y} \in(\text { layer 2) }
\end{array} \mathrm{i}=1,2\right. \\
\rho C_{p}(\boldsymbol{y})=\left[\begin{array}{c}
\rho_{m} C_{p m}, \text { if } \boldsymbol{y} \in \text { (layer 1) } \\
\rho_{f} C_{p f} \text { if } \boldsymbol{y} \in \text { (layer 2) }
\end{array}\right.
\end{gathered}
$$

The effective thermal properties for such structure are easily found by using standard heat conduction rules: $k_{11}^{*}=\frac{k_{m}+k_{f}}{2}, \frac{1}{k_{22}^{*}}=\frac{1}{2}\left(\frac{1}{k_{m}}+\frac{1}{k_{f}}\right)$. More generally, they are determined by computing first at the microscopic scale, the functions $w_{i}, i=1,2$, on the cell domain $\mathrm{Y}$, and by using the equation (9b). For this example, they are plotted on figure 3 and the numerical values which quantify the non isotropic property of the homogenized medium, are equal to:

$$
\begin{aligned}
\boldsymbol{K}^{*} & =\left[\begin{array}{ll}
k_{11}^{*} & k_{21}^{*} \\
k_{12}^{*} & k_{22}^{*}
\end{array}\right]=\left[\begin{array}{cc}
2.6 & 0 \\
0 & 0.38
\end{array}\right]\left(W \mathrm{~m}^{-1} \mathrm{~K}^{-1}\right) \\
\left(\rho C_{p}\right)^{*} & =\frac{1}{|Y|} \int_{Y} \rho C_{p} d y=0.683 * 10^{6}\left(\mathrm{~J} \mathrm{~m}^{-3} \mathrm{~K}^{-1}\right)
\end{aligned}
$$

Consequently, the characteristic times in the directions $\boldsymbol{e}_{1}, \boldsymbol{e}_{2}$, at the microscopic scale, are respectively: $\tau_{c, 1}=\frac{\left(\rho C_{p}\right)^{*} l^{2}}{k_{11}^{*}}=0.26 s ; \quad \tau_{c, 2}=\frac{\left(\rho C_{p}\right)^{*} l^{2}}{k_{22}^{*}}=1.80 \mathrm{~s}$

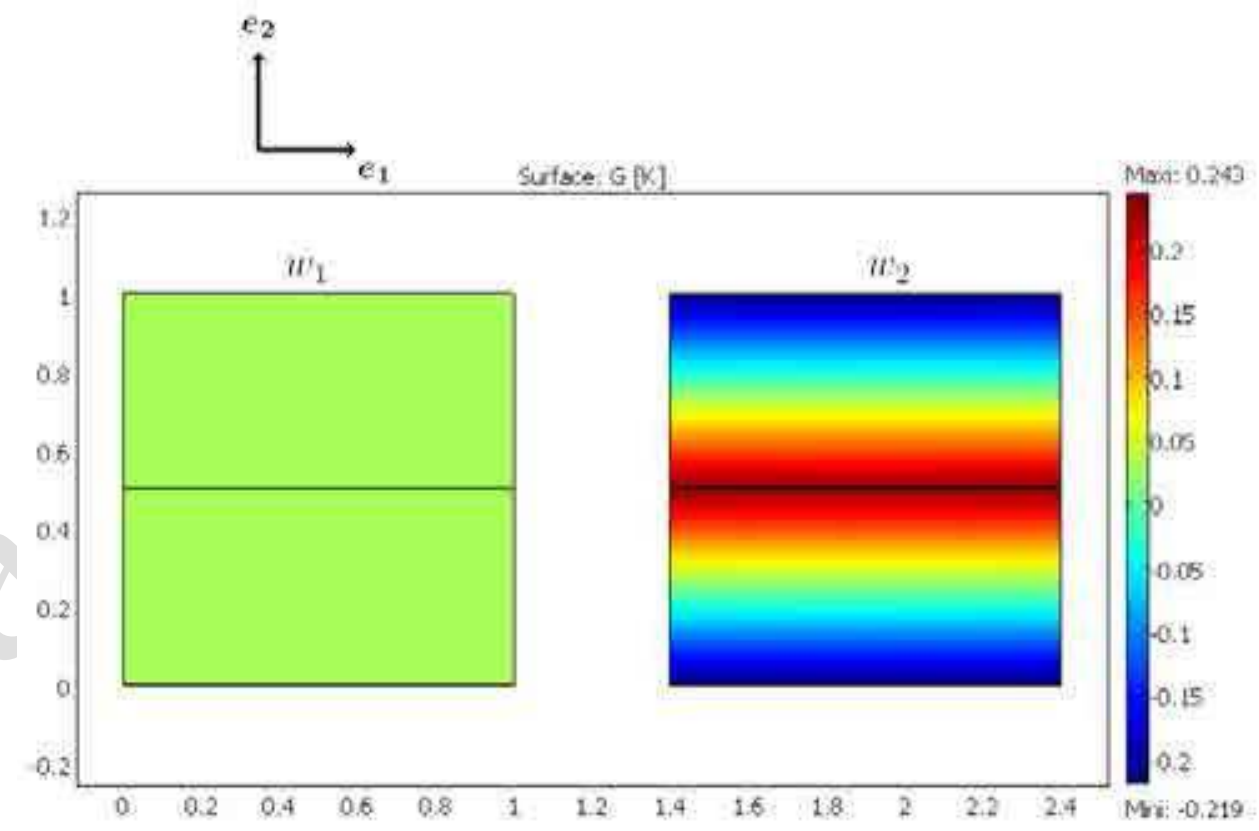

Figure 3: Numerical solutions $\omega_{i}(\boldsymbol{y}), \mathrm{i}=1,2$ computed on the periodic cell $Y$

Knowing the effective thermal properties, the solution $T^{\varepsilon}(x, t)$ of the transient heat conduction equation in the heterogeneous medium can be compared with the homogenized 
solution, given by the asymptotic expansion, equation (2). At the order $k=1$, the solution is $T^{0}(\boldsymbol{x}, \boldsymbol{y}, t)+\varepsilon T^{1}(\boldsymbol{x}, \boldsymbol{y}, t)$.

To capture "short time effects", the time step is chosen equal to $\Delta t=0.01 \mathrm{~s}$ up to $t=5 \mathrm{~s}$, and to $\Delta t=0.1 \mathrm{~s}$, up to the final time $t_{f}=90 \mathrm{~s}$. The solutions are compared on figures $4 \mathrm{a}-4 \mathrm{~b}$, at the locations $\left(x_{1}=5 \mathrm{~mm} ; x_{2}=5.3 \mathrm{~mm}\right)$ and $\left(x_{1}=5 \mathrm{~mm} ; x_{2}=5.8 \mathrm{~mm}\right)$. The plot shows clearly, for short times $(t<5 s)$, a difference between the heterogeneous and the homogenized solutions, while both solutions are quite similar for longer times.

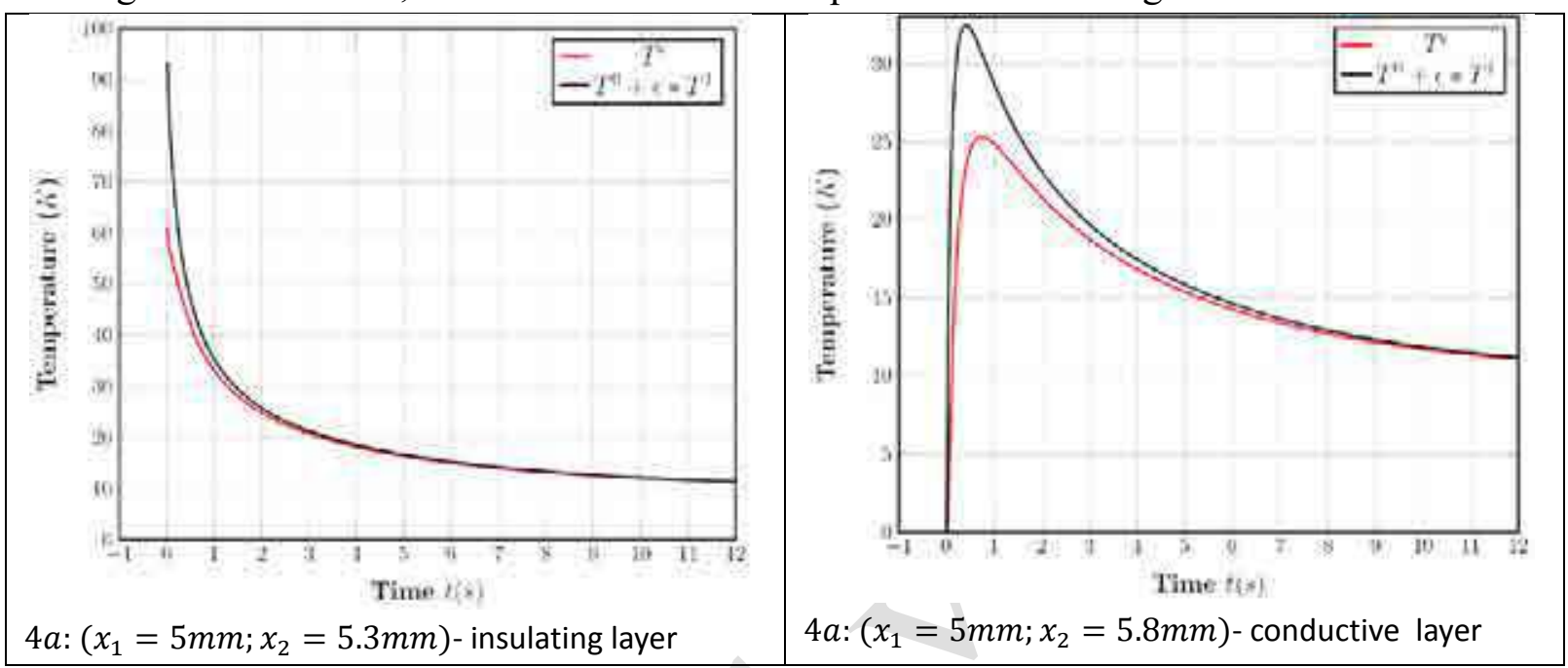

Figure 4: Comparison of the homogenized and heterogeneous temperatures- Case \#1.

It is observed that for short times (close to the initial time $t=0$ )

$$
T^{\varepsilon}(x, y, t) \neq T^{0}(x, t)+\varepsilon T^{1}(x, y, t)
$$

In fact, even if the field $T^{0}(\boldsymbol{x}, t=0)$ satisfies the initial condition $T^{\varepsilon}(\boldsymbol{x}, t=0)=T_{\text {ini }}(\boldsymbol{x})$, it is obvious in this example from the equations (5), (6), that it is not the case at the microscopic scale, especially when $T_{i n i}(\boldsymbol{x})$ is not uniform:

- Here, $\boldsymbol{\nabla}_{\boldsymbol{x}} T_{\text {ini }} \neq 0$ and $\omega_{2} \neq 0$ imply that $T^{1}(\boldsymbol{x}, \boldsymbol{y}, t=0) \neq 0$,

- it results that $T^{0}(\boldsymbol{x}, t=0)+\varepsilon \cdot T^{1}(\boldsymbol{x}, \boldsymbol{y}, t=0) \neq T_{\text {ini }}(\boldsymbol{x})$.

The deviation between $\left(T^{0}+\varepsilon T^{1}\right)$, the homogenized solution (at the order $k=1$ ) and the heterogeneous one $T^{\varepsilon}$, at short times, is not specific to this example, but is a consequence of the asymptotic expansion method. It is shown in the next section, how these "short time effects" can be corrected.

More generally, it means that the asymptotic expansion method developed above, provides a solution which satisfies the initial condition at the macroscopic scale, but does not guaranty that the initial condition of the functions $T^{k}(\boldsymbol{x}, \boldsymbol{y}, t=0), k \geq 1$, are equal to zero. 


\section{4.-Correction of the "short time effects "}

\section{1- Correcting terms}

In order to correct this problem of initial condition, correcting terms with an exponential time decreasing are introduced at short times. As in the spatial domain, a double asymptotic scale in the time range is considered [21-24], by introducing a "fast time" variable $\tau=t \cdot \varepsilon^{-2}$, and correcting terms, defined as the functions $T_{s t}^{k}(x, y, t, \tau), k \geq 1$, are added, leading to:

$$
T^{\varepsilon}(\boldsymbol{x}, \boldsymbol{y}, t, \tau)=T^{0}(\boldsymbol{x}, t)+\varepsilon\left[T^{1}(\boldsymbol{x}, \boldsymbol{y}, t)+T_{s t}^{1}(\boldsymbol{x}, \boldsymbol{y}, t, \tau)\right]+\cdots ; \quad \boldsymbol{x} \in \Omega, \boldsymbol{y} \in Y
$$

The use of a double time scale is not specific to this homogenization problem. More generally, it consists in introducing a new time variable $\tau$, such that $\tau=\frac{t}{\xi}$ with $\xi \ll 1$.

In 1-D transient heat conduction, at the macro-scale, we have $\frac{\partial T}{\partial t}=a \frac{\partial^{2} T}{\partial x^{2}}$ ( $a$ is the thermal diffusivity); by introducing the spatial variable $\boldsymbol{y}=\varepsilon^{-1} \boldsymbol{x}$ at the micro-scale, the equation can be written under the form: $\varepsilon^{2} \frac{\partial T}{\partial t}=a \frac{\partial^{2} T}{\partial y^{2}}$, which naturally introduces the new time variable $\tau=\frac{t}{\varepsilon^{2}}$, to get: $\frac{\partial T}{\partial \tau}=a \frac{\partial^{2} T}{\partial y^{2}}$.

Thus, the variable $\tau=\frac{t}{\xi}$ with $\xi=\varepsilon^{2} \ll 1$ is well convenient as a time variable at the micro-scale. The fact that $\xi=\varepsilon^{2}$ is very small allows us to consider that both time variables are (almost) independent and suggests to extend the asymptotic approach to the time interval, as it is done in the spatial domain.

By substituting this new asymptotic expansion in the heterogeneous heat conduction equation (1) we get a series of "time boundary layer" problems for each power of $\varepsilon$. At the order $k=$ 1 , according to developments similar to the double spatial scale approach [13], we obtain:

$$
T_{s t}^{1}(\boldsymbol{x}, \boldsymbol{y}, t, \tau)=\sum_{i=1}^{3} \frac{\partial T^{0}(\boldsymbol{x}, t)}{\partial x_{i}} z_{i}(\boldsymbol{y}, \tau)
$$

Consequently, the "short time" correcting term at the order $k=0$, for the approximation of the heat flux density can be expressed:

$$
\phi_{s t}^{0}(\boldsymbol{x}, \boldsymbol{y}, t, \tau)=\left[\boldsymbol{K}(\boldsymbol{y})\left(\boldsymbol{\nabla}_{\boldsymbol{y}} z_{i}(\boldsymbol{y}, \tau)\right)\right] \boldsymbol{\nabla}_{\boldsymbol{x}} T^{0}(\boldsymbol{x}, t)
$$

where the new variables $z_{i}(\boldsymbol{y}, \tau), i=1,2,3$ are the solutions of the transient periodic cell problems

$$
\left\{\begin{array}{c}
\left.\rho C_{p} \frac{\partial z_{i}(\boldsymbol{y}, \tau)}{\partial \tau}-\operatorname{div}_{y}\left[\boldsymbol{K}(\boldsymbol{y})\left(\boldsymbol{\nabla}_{\boldsymbol{y}} z_{i}(\boldsymbol{y}, \tau)\right)\right]=0 \text { in } Y \times\right] 0, t_{f}[ \\
z_{i}(\boldsymbol{y}, \tau=0)=-\omega_{i}(\boldsymbol{y}) \text { in } Y \\
z_{i} \text { periodic on } \partial Y
\end{array}\right.
$$




\section{2- Numerical example (Case \#2)- Correction of short time effects-}

The previous numerical example (case \#1) is thus continued. The function $\omega_{1}(\boldsymbol{y})$ is equal to zero in $Y$, hence the function $z_{1}(\boldsymbol{y}, \tau)=0$, too. The function $z_{2}(\boldsymbol{y}, \tau)$ is computed according to equation system (15).

The resulting first order correcting term $\delta^{1}(\boldsymbol{x}, t)=T_{s t}^{1}\left(\boldsymbol{x}, \boldsymbol{y}, t, \tau=t \varepsilon^{-2}\right)$, is plotted at different locations (figure 5). For practical reason, only the "slow" variable $t$ is chosen for plotting the time variations of $\delta^{1}(\boldsymbol{x}, t)$. It is observed that these terms tends towards zero whatever the location, but depending on the local thermal property (insulated or conductive layer), these terms take positive or negative values. Therefore, the homogenized solution without correcting terms $\left(T^{0}+\varepsilon . T^{1}\right)$ over- or underestimates the heterogeneous solution. Now the homogenized solutions $\left(T^{0}+\varepsilon\left[T^{1}+T_{s t}^{1}\right]\right)$ computed with the correcting terms $T_{s t}^{1}$, according to the new asymptotic expansion, are compared to the heterogeneous solutions on the figures 5a-5f. A very good agreement can be observed, whatever the spatial location. This numerical example illustrates the interest and the efficiency of the method.

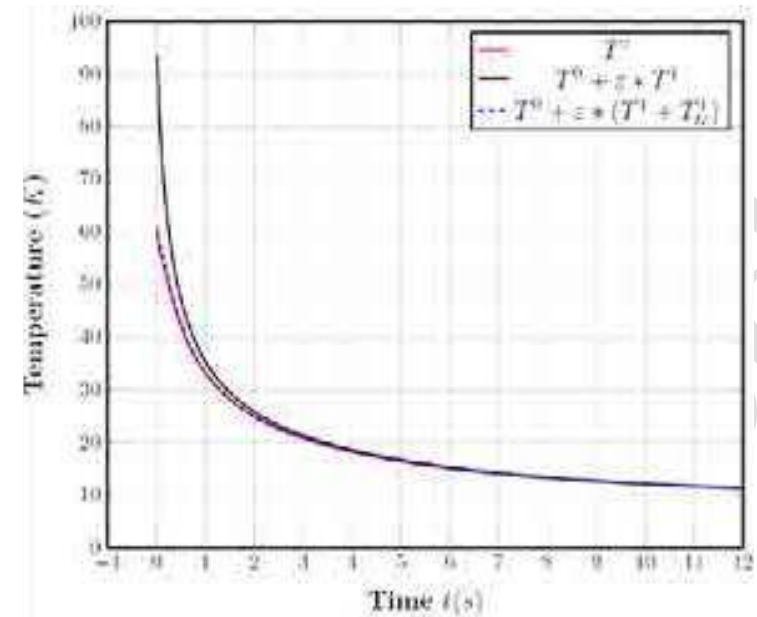

(a)

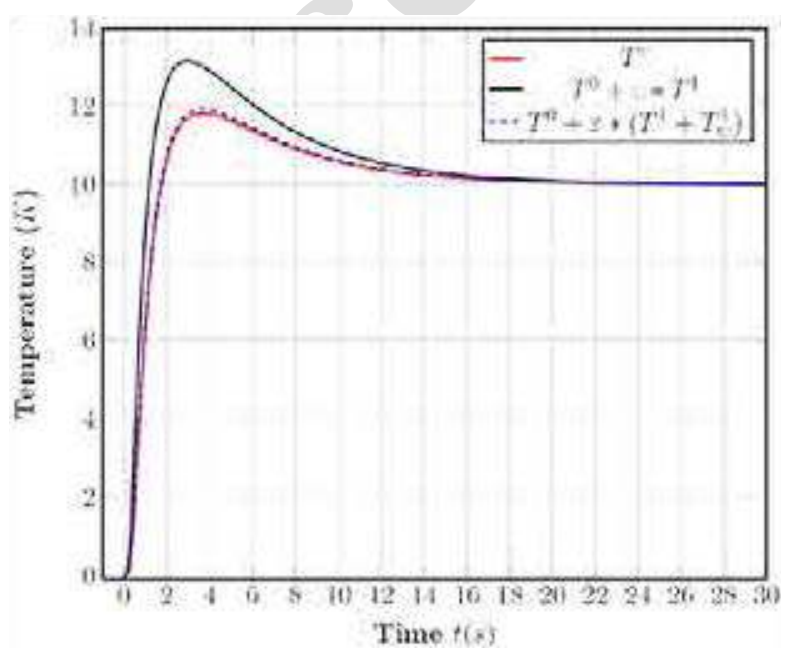

(c)

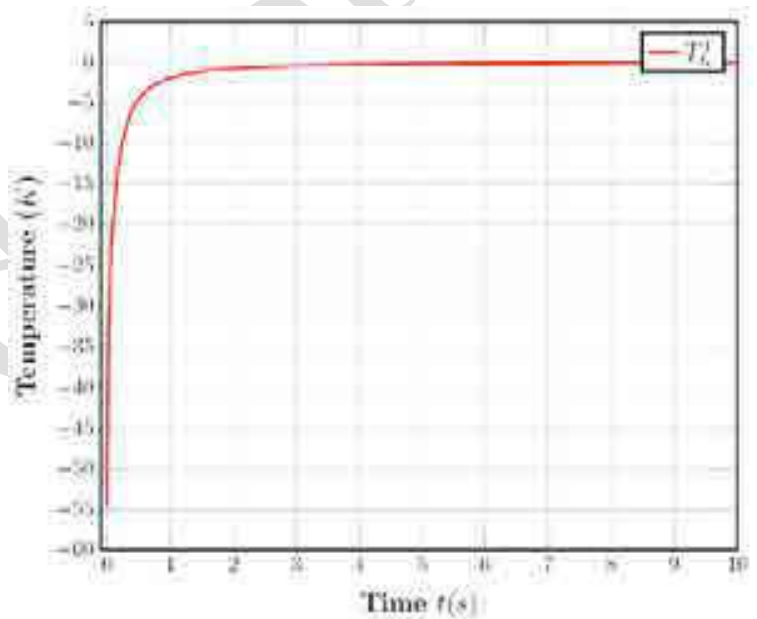

(b)

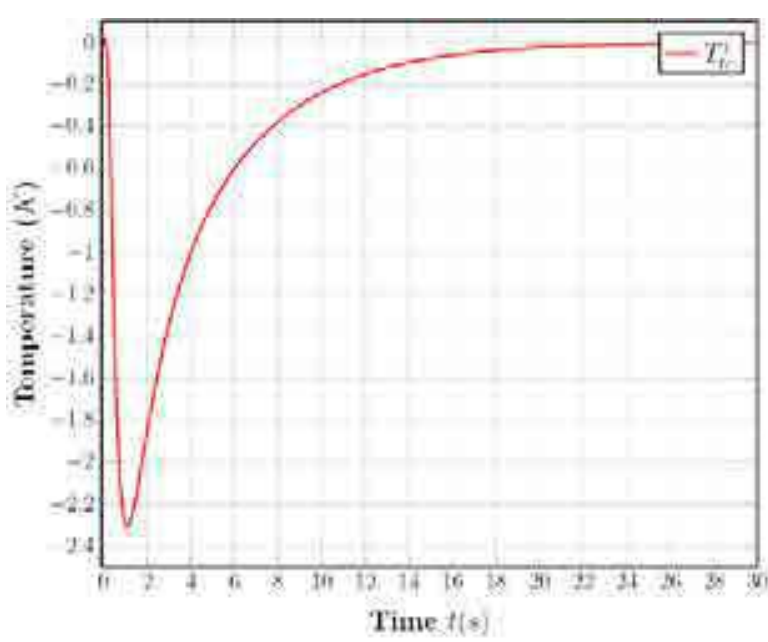

(d) 


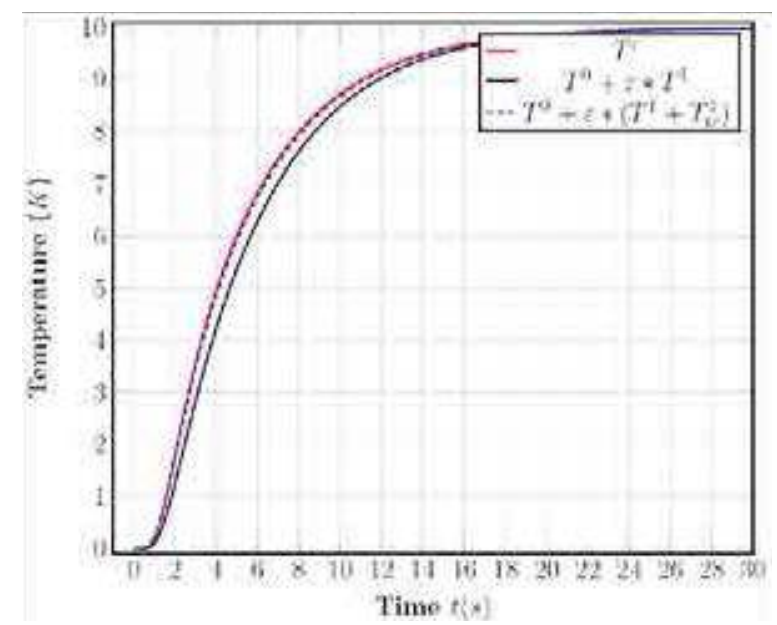

(e)

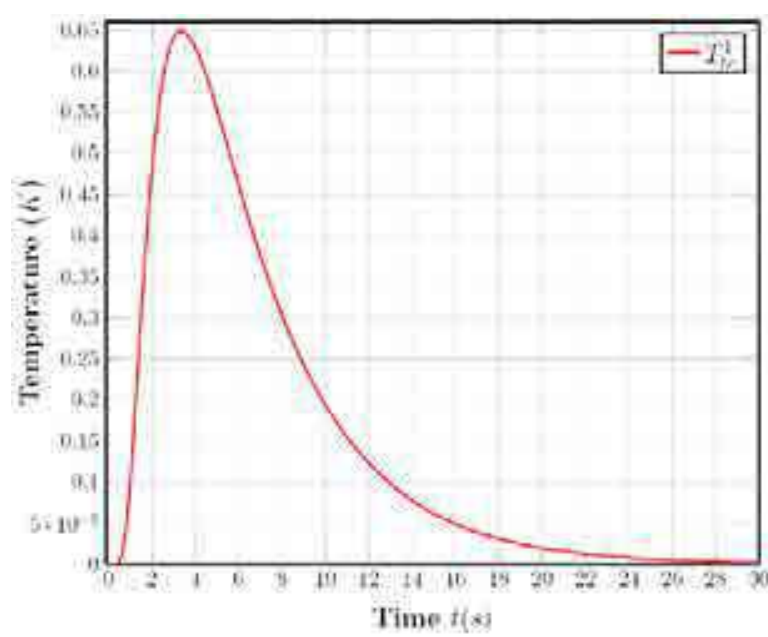

(f)

Figure 5: Comparison of the corrected homogenized and heterogeneous temperatures for different locations- Case \#2 / 5a-b: $\left(x_{1}=5 \mathrm{~mm} ; x_{2}=5.3 \mathrm{~mm}\right)$-; 5c-d: $\left(x_{1}=5 \mathrm{~mm} ; x_{2}=2.8 \mathrm{~mm}\right)$-; 5e-f: $\left(x_{1}=\right.$ $5 \mathrm{~mm} ; x_{2}=1.3 \mathrm{~mm}$ )

\section{5.- Correction of spatial edge effects}

\section{1- Correcting terms}

It was discussed in a previous work [13] of the authors, how periodic homogenization in steady state provides heat flux density $\phi^{0}$ and temperature $T^{0}$ fields, which are good approximations of the heterogeneous solutions $\phi^{\varepsilon}$ and $T^{\varepsilon}$ far enough from the boundary $\partial \Omega$ of the spatial domain. However, this approximation is not satisfactory anymore close to the boundary. This is first due to the loss of spatial periodicity. The second reason is that $\boldsymbol{\phi}^{\mathbf{0}}$ is generally not compatible with an arbitrary Neumann or Fourier conditions, since these conditions are only satisfied in a weak sense. Consequently, it is necessary to improve the accuracy of the homogenized solutions (temperature and/or heat flux) in the vicinity of the boundary. Correcting terms of spatial edge effects have thus to be determined.

Following the same approach than in the steady state analysis, a new asymptotic expansion is considered, which involves correcting terms at different order $T_{b l}^{k, m}, k \geq 1$. Their spatial variations are exponentially decreasing [19] and vanish far enough from the boundary $\Gamma_{j}$ :

$$
\begin{aligned}
& T^{\epsilon}=T^{0}(\boldsymbol{x}, t)+\varepsilon . {\left[T^{1}(\boldsymbol{x}, \boldsymbol{y}, t)+T_{s t}^{1}(\boldsymbol{x}, \boldsymbol{y}, t, \tau)+\sum_{m=1}^{3} T_{b l}^{1, m}(\boldsymbol{x}, \boldsymbol{y}, t, \tau)\right] } \\
&+\cdots ; \boldsymbol{x} \epsilon \Omega, \boldsymbol{y} \in Y
\end{aligned}
$$

The superscript values $m=1,2,3$ corresponds to one of the three different kinds of boundary condition usually associated to the heat conduction problem (see the set of equations (1)). The vicinity of the boundaries $\Gamma_{m=1,2,3}$ is defined as a strip $G_{m}$ into the spatial domain $\Omega$, in the normal direction to $\Gamma_{m}$, as illustrated on figure 6 . The surface of $G_{m}$ is denoted $\Gamma_{m}^{\prime}$ and $\Gamma_{m}^{\prime} \subset \Gamma_{m}$ : 


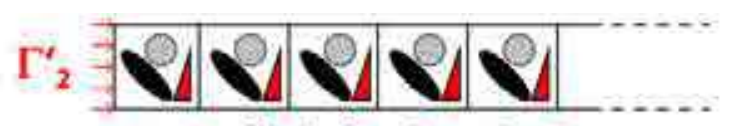

(ii) semi-infinite domain $C_{2}$

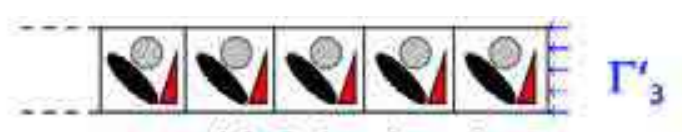

(b) semi-infinite domain $G_{3}$

Figure 6: Presentation of the sub-domains $\mathrm{G}_{\mathrm{m}}$ into the spatial domain $\Omega$, in the normal direction $\left(\boldsymbol{e}_{\mathbf{1}}\right)$ to the boundaries $\Gamma_{\mathrm{m}}$

In this example, both boundaries $\Gamma_{m=2}$ (Neumann condition) and $\Gamma_{m=3}$ (Dirichlet condition) are normal to the $\boldsymbol{e}_{\mathbf{1}}$ direction. Each correcting term $T_{b l}^{1, m}$ is defined for $x \in \Gamma_{m}, m=2$ or 3 , and $\boldsymbol{y}=\left(y_{1}, y_{2}\right) \in Y$. It is periodic in the $\boldsymbol{e}_{2}$ direction and exponentially decreasing along $\boldsymbol{e}_{\mathbf{1}}$.

By substituting this new asymptotic expansion in the heat conduction equation and by identifying the terms for the different powers of $\varepsilon$, we get a series of "spatial boundary layer" problems. At the order $k=1$, it comes:

$$
T_{B L}^{1, m}(\boldsymbol{x}, \boldsymbol{y}, t, \tau)=\sum_{i=1}^{3} \frac{\partial T^{0}}{\partial x_{i}}(\boldsymbol{x}, t) \chi_{i}^{m}(\boldsymbol{y}, \tau)
$$

The «short time » effects have to be taken into account over the entire spatial domain, thus even in the vicinity of the boundaries. Then the correcting term $T_{B L}^{1, m}(\boldsymbol{x}, \boldsymbol{y}, t, \tau)$ a priori depends of both time variables $t$ and $\tau$.

Consequently, the "edge effect" correcting term at the order $k=0$, for the approximation of the heat flux density can be written:

$$
\phi_{b l}^{0, m}(x, y, t, \tau)=\left[K(y) \nabla_{y} \chi^{m}(\boldsymbol{y}, \tau)\right] \boldsymbol{\nabla}_{\boldsymbol{x}} T^{0}(\boldsymbol{x}, t)
$$

The functions $\chi_{i}^{m}(\boldsymbol{y}, \tau), i=1$ to $3 ; m=1$ to 3 , are the solutions of the following set of equations:

$$
\left\{\begin{array}{c}
\left.\rho C_{p}(\boldsymbol{y}) \frac{\chi_{i}^{m}(\boldsymbol{y}, \tau)}{\partial \tau}-\operatorname{div}_{\boldsymbol{y}}\left[\boldsymbol{K}(\boldsymbol{y})\left(\nabla_{\boldsymbol{y}} \chi_{i}^{m}(\boldsymbol{y}, \tau)\right)\right]=0 \text { in } G_{m} \times\right] 0, t_{f}[ \\
\chi_{i}^{m}(\boldsymbol{y}, \tau=0)=-\omega_{i}(\boldsymbol{y})-z_{i}(\boldsymbol{y}, \tau=0) \text { in } G_{m} \\
\quad+\text { Boundary Condition on } \Gamma_{m}^{\prime} \\
\chi_{i}^{m} \text { periodic in the } \boldsymbol{e}_{2}, \boldsymbol{e}_{3} \text { direction }
\end{array}\right.
$$

Like in the steady state analysis [13], depending on the condition chosen for determining the temperature field on the boundary $\Gamma_{m}$ (Fourier, Neumann or Dirichlet conditions), the boundary condition for computing $\chi_{i}^{m}$ on $\Gamma_{m}^{\prime}$ takes different forms on $\left.\Gamma_{m}^{\prime} \times\right] 0, t_{f}[$ :

- Neumann or Fourier conditions ( $m=1$ or 2$)$ :

$$
\begin{aligned}
\boldsymbol{K}(\boldsymbol{y})\left(\boldsymbol{\nabla}_{\boldsymbol{y}} \chi_{i}^{m}(\boldsymbol{y}, \boldsymbol{\tau})\right) \cdot \boldsymbol{n} & \\
= & -\boldsymbol{K}(\boldsymbol{y})\left(\left(\boldsymbol{e}_{\boldsymbol{i}}+\boldsymbol{\nabla}_{\boldsymbol{y}} \omega_{i}(\boldsymbol{y})+\boldsymbol{\nabla}_{\boldsymbol{y}} z_{i}(\boldsymbol{y}, \tau)\right)\right) \cdot \boldsymbol{n} \\
& +\frac{1}{|Y|} \int_{Y} \boldsymbol{K}(\boldsymbol{y})\left(\boldsymbol{e}_{\boldsymbol{i}}+\boldsymbol{\nabla}_{\boldsymbol{y}} \omega_{i}(\boldsymbol{y})\right) d Y . \boldsymbol{n}
\end{aligned}
$$

- Dirichlet condition $(m=3)$ :

$$
\chi_{i}^{m}(\boldsymbol{y}, \tau)=-\omega_{i}(\boldsymbol{y})-z_{i}(\boldsymbol{y}, \tau)
$$

Furthermore, we can determine the depth of the "heat conduction boundary layer" in steady state by solving numerically a specific eigenvalues problem set on $G_{m}$, depending on the kind 
of the boundary condition on $\Gamma_{m}$. For the above example, in the vicinity of $\Gamma_{m=1}$ the exponential decreasing in space of $T_{b l}^{1, m=1}$ in the $\boldsymbol{e}_{\mathbf{1}}$ direction, can be written [19]:

$$
\chi_{i}^{m}(\boldsymbol{y})=\Psi_{i}^{m}(\boldsymbol{y}) \cdot e^{-\delta_{m} y_{1}}
$$

where the parameter $\delta_{m}$ is given by the lowest solution of an eigenvalues problem and $\Psi_{i}^{m}(\boldsymbol{y})$ is the associated eigenvector on the sub-domain $G_{m}$. Hence the depth $d_{m}$ of the spatial boundary layer close to $\Gamma_{m}$ where the correcting term $T_{b l}^{1, m}$ does not spatially vanish can be estimated by $d_{m} \approx \frac{3}{\delta_{m}}$. More mathematical developments and numerical results can be found in [13].

In practice, the sub-domain $G_{m}$ where $\chi_{i}^{m}(\boldsymbol{y}, \tau)$ is computed, is truncated at the distance $d_{m}$ from $\Gamma_{\mathrm{m}}^{\prime}$ along the $\boldsymbol{e}_{\mathbf{1}}$ direction, according to the exponential decreasing property of $\chi_{i}^{m}(y, \tau)\left(\chi_{i}^{m}\right.$ tends towards zero when $\left.y_{1} \rightarrow \infty\right)$. The Dirichlet condition $\chi_{i}^{m}\left(y_{1}=d_{m}, \tau\right)=0$, is thus naturally chosen, with $d_{m}$ large enough, see [13].

\section{2- Numerical example (Case \#3)- Correcting edge effects due to Neumann condition -}

To illustrate the interest in determining the correcting terms $T_{b l}^{1, m}$ associated to a Neumann boundary condition $(m=2)$, the previous example is continued, with the following data (see figure 7):

- The initial condition is isothermal $T_{i n i}(x)=0$,

- The heat flux density on the front face is assumed to be uniform and fixed (Neumann boundary condition) to a constant value: $F(t)=2.10^{3} \mathrm{~W} \mathrm{~m}^{-2}$,

- The temperature is imposed on the back face (Dirichlet boundary condition),

- Periodic conditions are considered on the other boundaries, to avoid "corner effects". It can be observed on the plots depicted in figures $8 \mathrm{a}-8 \mathrm{~d}$, that the resulting correcting term $T_{B L}^{1, m}$ vary both in space and time. The homogenized solution is plotted on the front face $\left(x_{1}=\right.$ $0)$, in the insulating layer $\left(x_{2}=5.3 \mathrm{~mm}\right)$ and in the conductive layer $\left(x_{2}=5.8 \mathrm{~mm}\right)$. Without correcting term, these plots show a strong deviation with the heterogeneous solution in the insulating layer, while the agreement with the corrected homogenized solution is quite good.

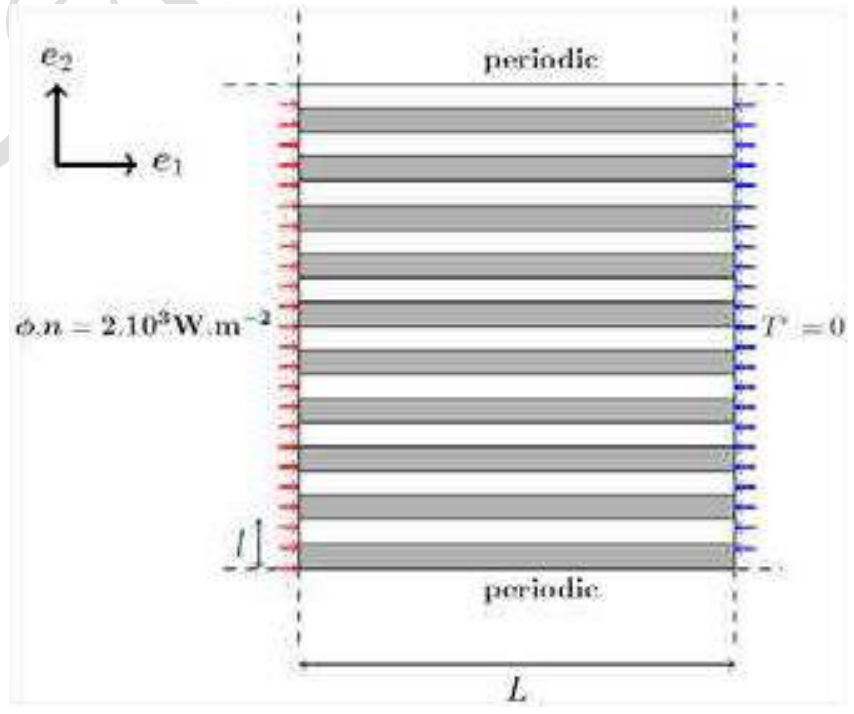

Figure 7: Presentation of the multi-layered medium and the periodic cell - Case \#3: isothermal initial condition together with a Neumann and a Dirichlet boundary conditions. 


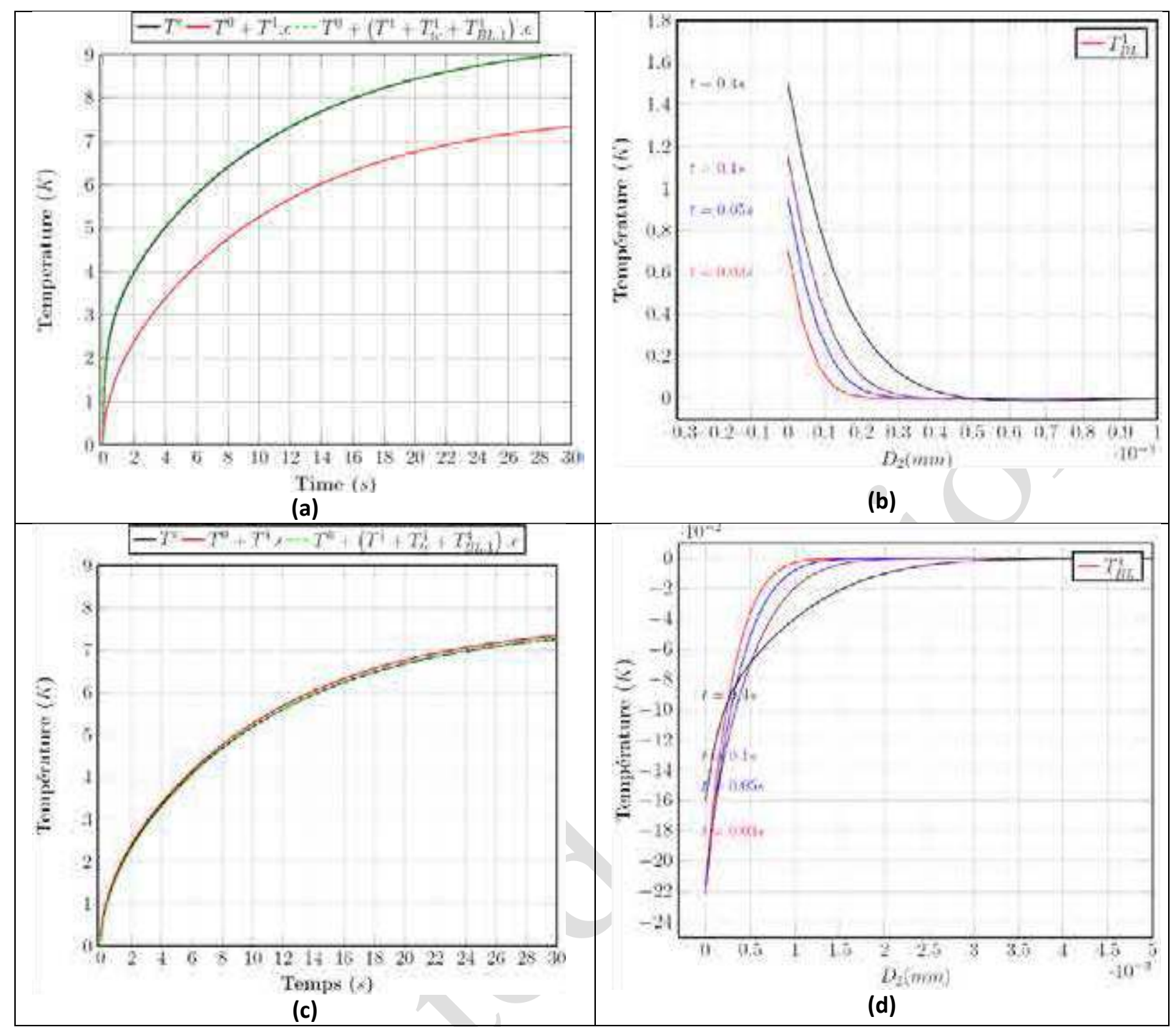

Figure 8: (8a)-(8c) Heterogeneous and corrected homogenized temperatures on the front face $\left(x_{1}=0\right)$; (8b)-(8d) Spatial variations of the correcting terms close to the front face in the $\boldsymbol{e}_{\mathbf{1}}$ direction -Case \#3; (8a)-(8b) $x_{2}=5.3 \mathrm{~mm}$, insulating layer; (8c)-(8d) $x_{2}=5.8 \mathrm{~mm}$, conductive layer

The correcting terms $T_{B L}^{1, m}$ are still varying in space from the boundary $\left(x_{1}=0\right)$; their profiles are exponentially decreasing in space whereas they increase in time up to a stationary solution ( $\mathrm{t}>0.3 \mathrm{~s})$. The spatial depth is lower than the value $\left(d_{m=2}=0.5 \mathrm{~mm}\right)$ predicted by the solution of the eigenvalues problem in the stationary study. The correcting terms which result of the Dirichlet condition on the back face can be neglected. More details are given in [13].

\section{6.-Application to the modeling of a heat pulse response into a periodic medium-}

The laser flash [3] device is used to perform classical thermal experiments aiming to determine experimentally the effective thermal properties of materials, heterogeneous or not. The data processing is based on the inverse analysis of measurements of the temperature rises which result of a heat pulse on a face of the sample. When these experiments are performed in order to characterize heterogeneous structures, the homogenized solutions of the transient heat conduction problem have to be compared to the measurements (instead of the heterogeneous 
solution). Then it is important to take into account these «short time » and «spatial edge » effects in the heat transfer modeling of the experiment (or at least to keep them in mind) to avoid biased estimation. In this section we perform the inverse analysis of such numerical experiments to illustrate the bias which would exist on the determination of the components of the thermal diffusivity tensor, when these effects are neglected.

\section{1- The numerical measurements from the "heterogeneous solution" (Case \#4)}

The numerical example of the bi-layered structure is continued as sketched in figure 9, but new data given in table 2 are considered, as discussed in the work of Fudym et al [11]. The heat pulse (duration $=0.001 \mathrm{~s}$ ) is imposed at the front face of a rectangular sample (the layers are normal to the $\boldsymbol{e}_{2}$ direction). Its thickness is $e=5 \mathrm{~mm}$.

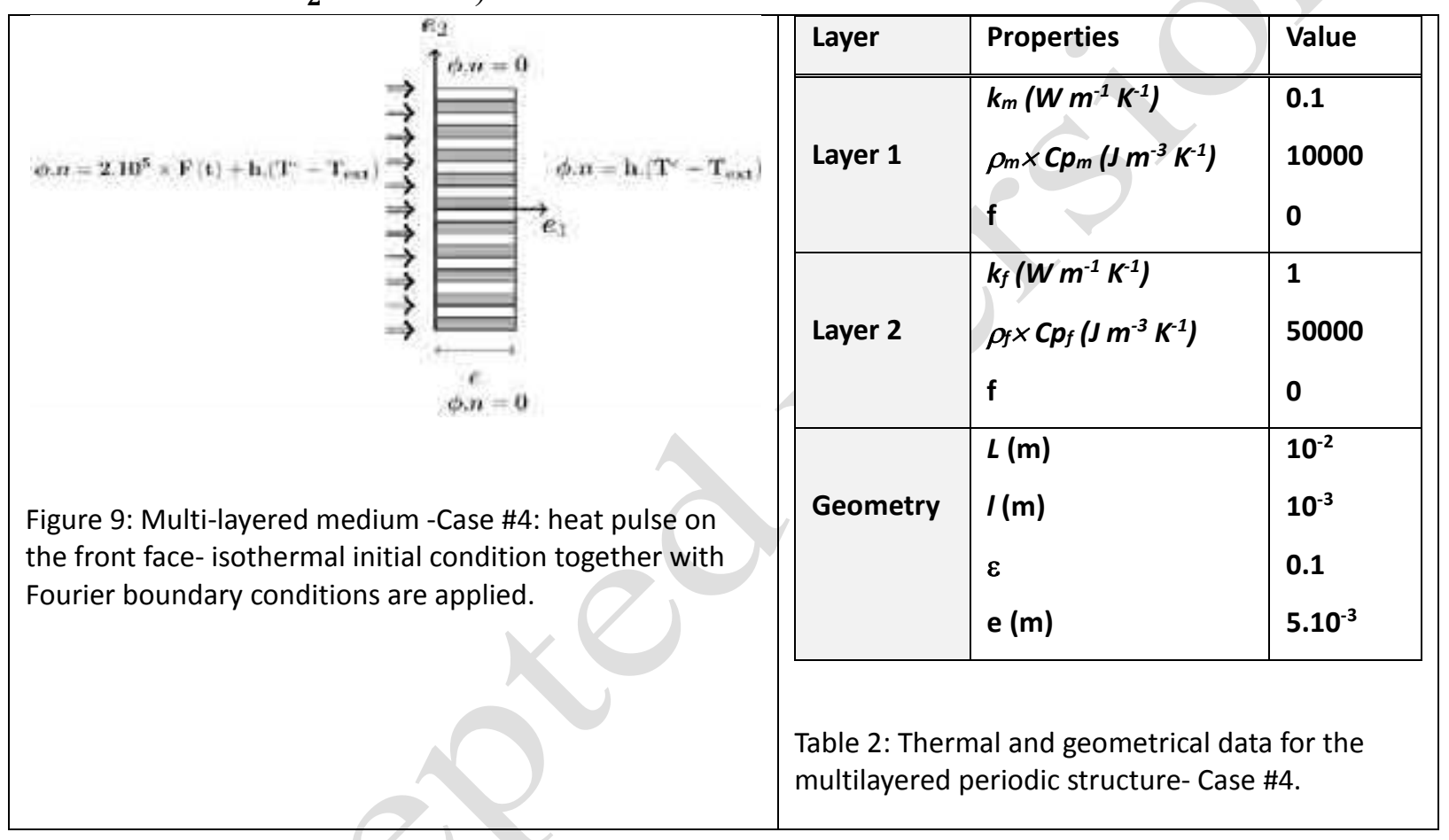

The initial state is supposed to be uniform, $T_{i n i}(\boldsymbol{x})=0$. The heat losses are modeled by a Fourier condition on the front and back faces with the external temperature $T_{\text {ext }}=0$ and a heat transfer coefficient $h=100 W \cdot m^{-2} \cdot K^{-1}$. Of course, this value should be not so high in practice, but it has been chosen here to emphasize the "edge effects". For realistic values of the coefficient $h$ (for example $h=10 \mathrm{~W} \cdot \mathrm{m}^{-2} \cdot \mathrm{K}^{-1}$ ), the magnitude of the correcting terms $T_{b l}^{1, m}$ are much smaller. Adiabatic condition is assumed on the others boundaries.

The resulting measurements are simulated on the back face. They are obtained by computing the solution $T^{\varepsilon}(\boldsymbol{x}, t)$ of the heat equation on the heterogeneous domain and plotted on the figures $10 a-b$. 


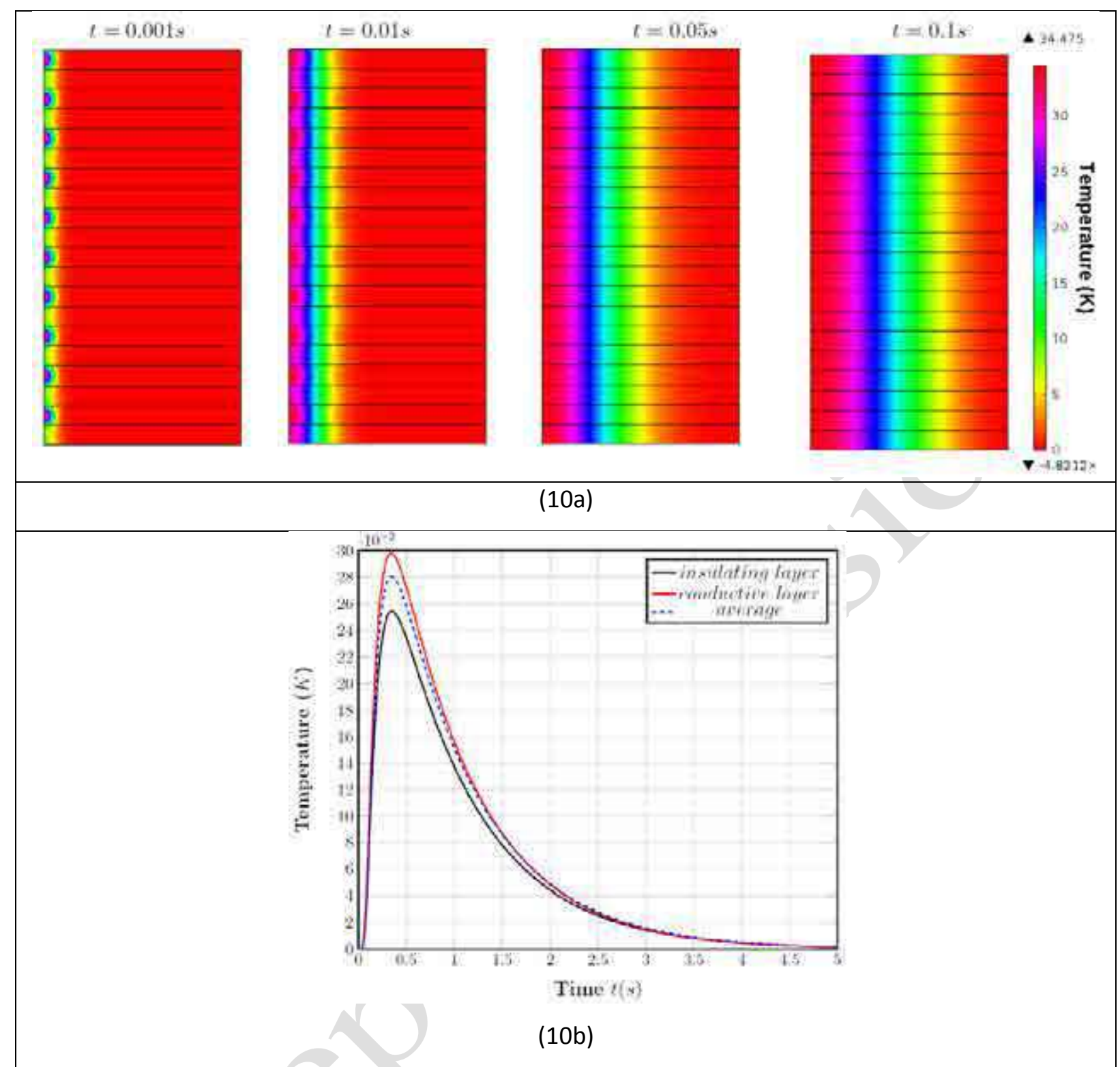

Figure 10: Heterogeneous temperatures $T^{\varepsilon}(x, t)$ computed for a laser flash experiment- Case \#4. (10.a): inside the slab, at short times : $t=0.001,0.01,0.05,0.1$ s. (10.b) on the back face, at different locations.

We observe that the temperature rise depends on the location of the sensor on the back face of the sample. The maximal temperature deviation in the insulated layer reaches $15 \%$ with respect to that of the conductive layer. 


\section{2- The temperature response with the space-time homogenized method.}

The effective thermal properties computed with the data given in table 2, are calculated:

- conductivity tensor : $\boldsymbol{K}^{*}=\left[\begin{array}{ll}k_{11}^{*} & k_{21}^{*} \\ k_{12}^{*} & k_{22}^{*}\end{array}\right]=\left[\begin{array}{cc}0.550 & 0 \\ 0 & 0.182\end{array}\right]\left(W m^{-1} K^{-1}\right)$; and $\kappa=k_{f} / k_{m}=$ 10

- heat capacity: $\left(\rho C_{p}\right)^{*}=\int_{Y} \rho C_{p} d y=3 * 10^{4} \mathrm{~J} \mathrm{~m}^{-3} K^{-1}$; and $\rho_{f} C_{p f} / \rho_{m} C_{p m}=5$

- diffusivity components: $a_{/ /}^{*}=18.3 * 10^{-6} \mathrm{~m}^{2} . \mathrm{s}^{-1} ; a_{\perp}=5.5 * 10^{-6} \mathrm{~m}^{2} \mathrm{~s}^{-1}$

and the characteristic times in the directions $\boldsymbol{e}_{1}, \boldsymbol{e}_{2}$, at the microscopic scale, are respectively:

$$
\tau_{c, 1}=\frac{\left(\rho C_{p}\right)^{*} l^{2}}{k_{11}^{*}}=0.0545 s ; \quad \tau_{c, 2}=\frac{\left(\rho C_{p}\right)^{*} l^{2}}{k_{22}^{*}}=0.165 \mathrm{~s}
$$

The homogenized solutions on the back face, computed with and without first order correcting terms $T_{b l}^{1, m=1}$, from the multi-scale space-time expansion method (equations (16)), are shown on figures 11.a (insulating layer) and 11.b (conductive layer). The temperatures are compared to the heterogeneous solutions (see also figure 10.b). The discrepancies observed without $T_{b l}^{1, m=1}$ (red lines) are well corrected when this term is considered (blue lines), whatever the sensor location within the insulating or the conductive layer.

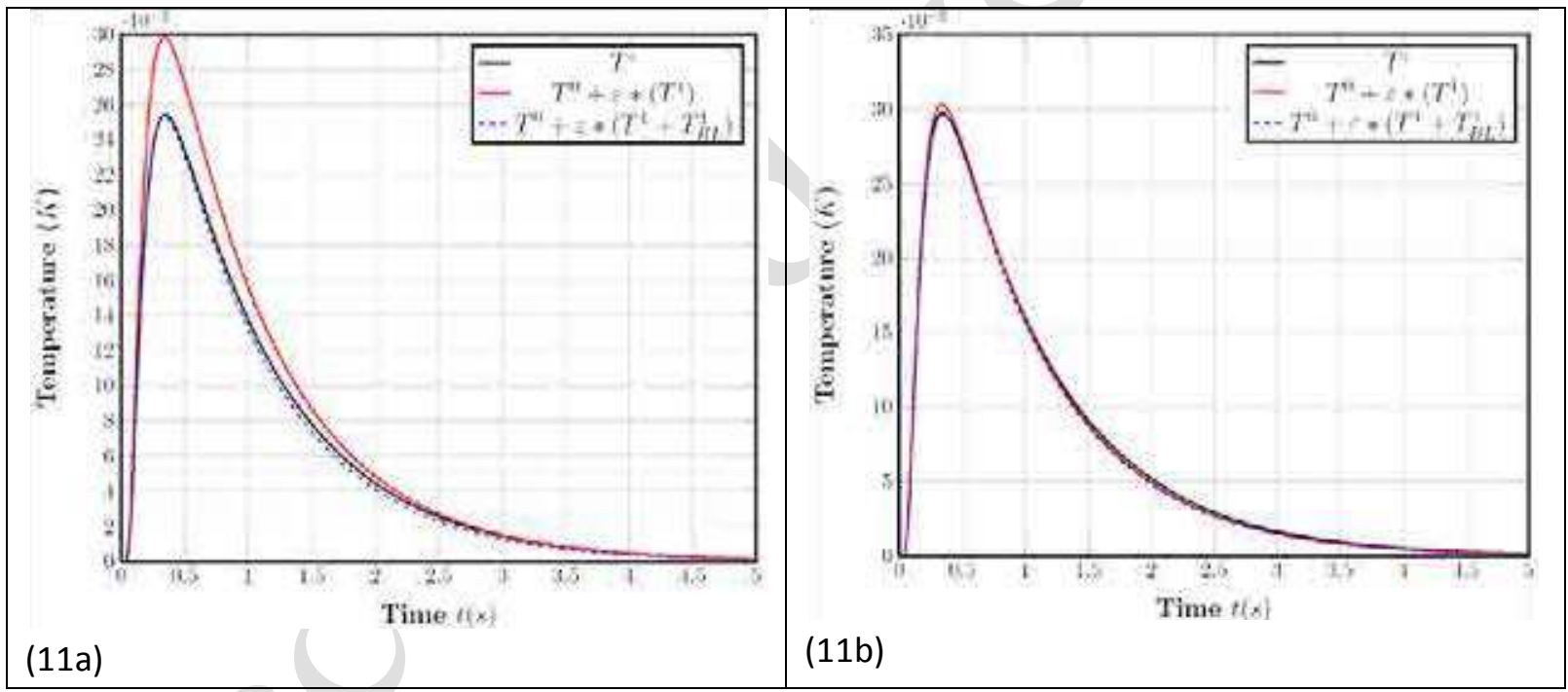

Figure 11: Homogenized temperatures on the back face computed with and without the correcting terms and compared to the heterogeneous solution- Case \#4. (11a): insulating layer $\left(x_{2}=5.3 \mathrm{~mm}\right)$, (11b) conductive layer at $\left(x_{2}=5.8 \mathrm{~mm}\right)$.

The homogenized heat flux density component $\boldsymbol{\phi}^{\mathbf{0}} . \boldsymbol{e}_{\mathbf{1}}$ computed on the back face is plotted on figures $12 \mathrm{a}-\mathrm{b}$ and compared to the heterogeneous solution $\boldsymbol{\phi}^{\boldsymbol{\varepsilon}} \cdot \boldsymbol{e}_{\mathbf{1}}$. Without corrected terms, the homogenized solutions are underestimated in the insulating layer $\left(x_{2}=5.3 \mathrm{~mm}\right)$, while it is overestimated in the conductive layer $\left(x_{2}=5.8 \mathrm{~mm}\right)$.

The corrected solution $\boldsymbol{\phi}^{\mathbf{0}}+\boldsymbol{\phi}_{\boldsymbol{B} \boldsymbol{L}}^{\mathbf{0}}$ is almost identical to $\phi^{\varepsilon}$ in the conductive layer (figure 12b), whereas a deviation still exist in the insulating layer (figure 12a). It reaches a maximum at short times $(t=0.5 \mathrm{~s})$ and finally tend towards zero further. In fact, the figures $12 \mathrm{c}$ and $12 \mathrm{~d}$ illustrate how both components of the heat flux density $\phi^{\varepsilon}$ at $t=0.5 \mathrm{~s}$ are not spatially uniform, and how the correcting terms $\phi_{B L}^{0}$ allows to take into account this non uniformity. 


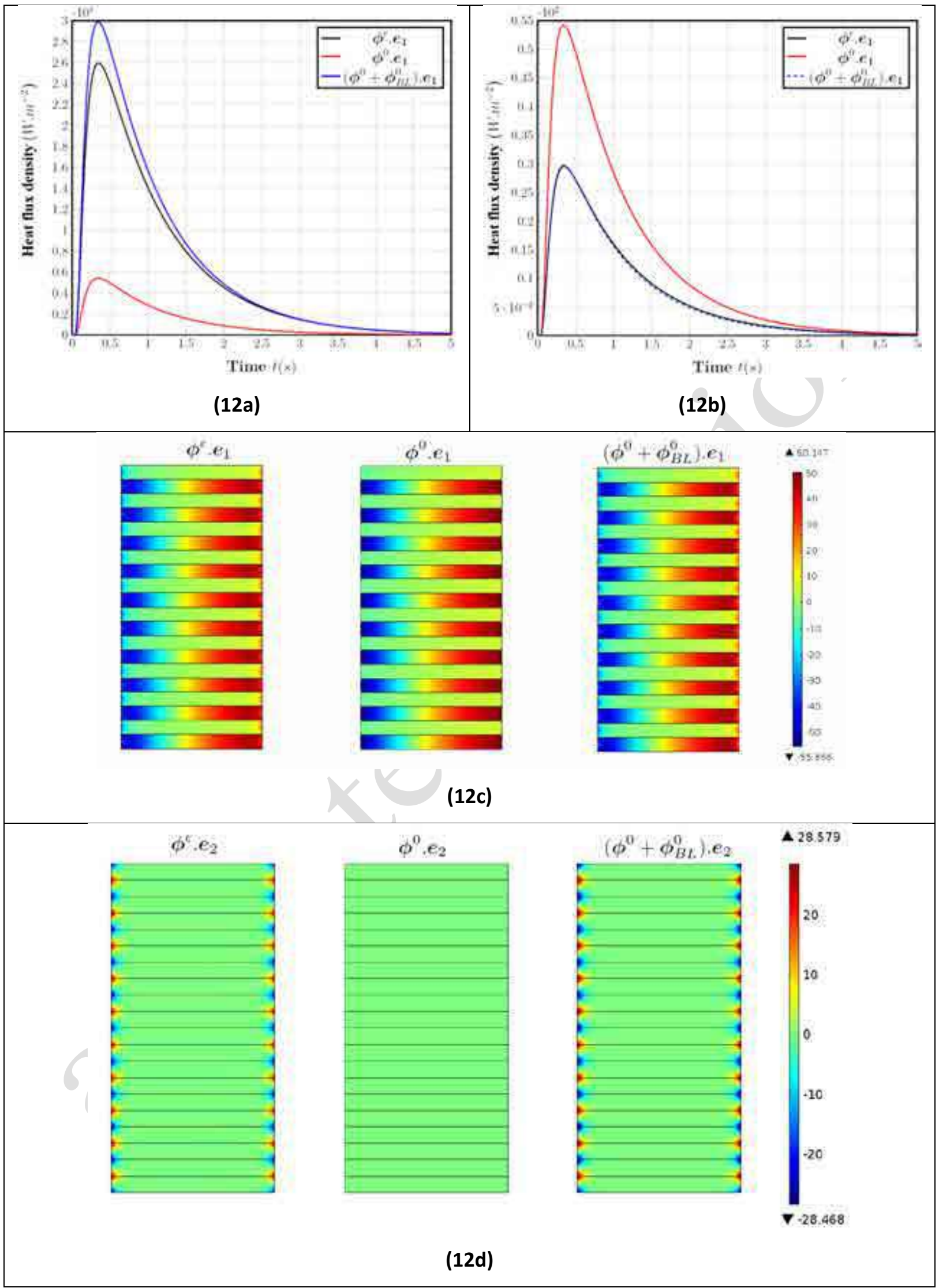

Figure 12: Homogenized heat flux density on the back face, computed without and with the correcting terms compared to the heterogeneous solution- Case \#4. (12a) at $x_{2}=5.3 \mathrm{~mm}$, insulating layer, $(12 \mathrm{~b})$ at $x_{2}=5.8 \mathrm{~mm}$ conductive layer, (12c) heat flux density component in the direction $\boldsymbol{e}_{1}$, at $t=0.5 \mathrm{~s},(12 \mathrm{~d})$ heat flux density component in the direction $\boldsymbol{e}_{2}$, at $t=0.5 \mathrm{~s}$. 


\section{3- The biased estimation of the thermal diffusivity}

In the LF experiment, the value of the effective thermal diffusivity component $a_{/ /}$of the heterogeneous medium is estimated by matching the back face temperature measurements to the response of a homogenized heat conduction model. If no correcting terms (especially due to edge effects) are considered in the homogenized solution, a modeling error is done as illustrated above, then the matching process will give a biased estimation value $a_{/ /}^{e s t}$.

To evaluate this bias, the above numerical experiment is continued. Following the asymptotic expansion method, the "true" value of the effective thermal diffusivity $a_{/ /}$of the homogenized medium is thus equal to $a_{/ /}^{*}=18.3 * 10^{-6} \mathrm{~m}^{2} . \mathrm{s}^{-1}$

The matching process which aims to get $a_{/ /}^{e s t}$ from measurement data $Z(t)$ is used in order to observe the influence on the location $x_{2}$ of the thermal sensor or IR detector, on the back face, in the three following cases:

- $x_{2}=5.3 \mathrm{~mm}$, sensor located in the layer $\# 1, Z(t)=T^{\varepsilon}\left(e, x_{2}, t\right)$

- $x_{2}=5.8 \mathrm{~mm}$, sensor located in the layer $\# 2, Z(t)=T^{\varepsilon}\left(e, x_{2}, t\right)$

- (IR detector) average value over the back face, $Z(t)=\frac{1}{l} \int_{0}^{l} T^{\varepsilon}\left(e, x_{2}, t\right) d x_{2}$

These three thermal responses $Z(t)$ are plotted on the figures $10 \mathrm{~b}$. They are the heterogeneous solution $T^{\varepsilon}\left(e, x_{2}, t\right)$ which have been first computed on the back face (section 6.1) with the data given in table 2 .

Details of the matching process can be found in [3]. It allows for each thermal response $Z(t)$ to determine the parameter $a_{/ /}^{e s t}$ for which the homogenized response $Z_{\text {mod }}(t)$ gives the best fit: $Z(t) \approx Z_{\text {mod }}(t)=T_{\text {exact }}\left(x=e, t ; a_{/ /}^{e s t}\right)$. The solution $T_{\text {exact }}$ is recalled in appendix 1 . The temperature residual errors $Z(t)-Z_{\text {mod }}(t)$ are plotted in figures 13a-c.

The three estimated values $a_{/ /}^{e s t}$ of the thermal diffusivity component $a_{/ /}$and the resulting bias $\Delta a=\left|a_{/ /}^{*}-a_{/ /}^{e s t}\right|$ are summarized in table 3 .

\begin{tabular}{|c|c|c|c|}
\hline Sensor location & $\begin{array}{l}\text { Estimated value } \\
a_{/ /}^{e s t}\left(m^{2} s^{-1}\right)\end{array}$ & $\begin{array}{c}\text { Bias } \\
\Delta a=\left|a_{/ \mid}^{*}-a_{/ \mid}^{e s t}\right|\end{array}$ & $\frac{\Delta a}{a_{/ /}^{*}}$ \\
\hline Insulating Layer \#1 & 1.692.e-5 & $0.138 . e-5$ & $7.54 \%$ \\
\hline Conductive Layer \#2 & $1.826 . \mathrm{e}-5$ & $0.028 . e-5$ & $1.55 \%$ \\
\hline Average response & $1.781 . e-5$ & $0.073 . e-5$ & $3.99 \%$ \\
\hline
\end{tabular}

Table 3: Biased estimations of the thermal diffusivity component $a_{/ /}$- Influence of the sensor location- Case \#4 


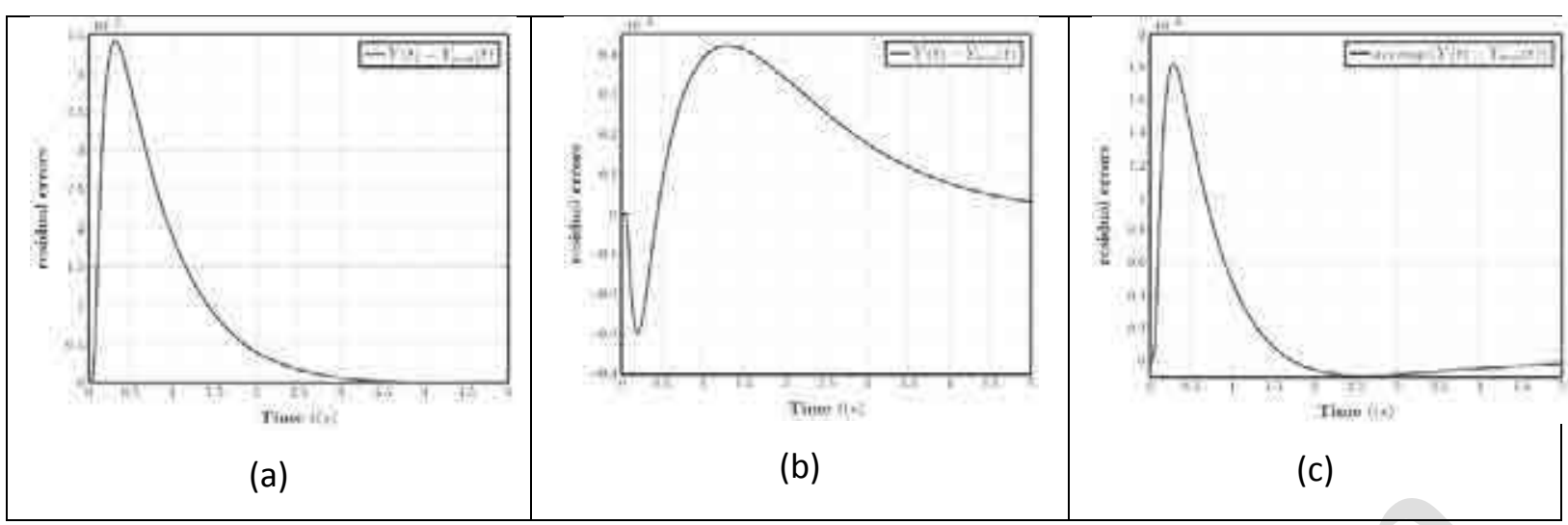

Figure 13: Temperature residual errors resulting of the data matching process on the back face - Case \#4 / -Influence of the sensor location. (a) insulating layer $\left(\mathrm{x}_{2}=5.3 \mathrm{~mm}\right),(\mathrm{b})$ conductive layer $\left(\mathrm{x}_{2}=\right.$ $5.8 \mathrm{~mm}),(\mathrm{c})$ average response.

To conclude about these numerical experiments, let us underlined three main results:

- the homogenized solution without correcting terms is uniform on the back face and is identical to the homogeneous solution $T_{\text {exact }}$ computed with $a_{/ /}=a_{/ /}^{*}$, the "true" value determined from the effective properties

- the homogenized responses computed with the correcting terms $T_{b l}^{1, m}$ (and the "true" value $a_{/ /}^{*}$ ) are quite identical for the three cases to the data $Z(t)=T^{\varepsilon}(t)$, given by the heterogeneous model, and shown on figures $10 \mathrm{~b}$.

- the homogeneous solutions $T_{\text {exact }}$ (without correcting terms $T_{b l}^{1, m}$ ), fit rather well the heterogeneous ones $T^{\varepsilon}$ only if the thermal diffusivity component $a_{/ /}^{e s t}$ is chosen depending on the sensor location on the back face, which has no physical meaning.

These results confirm the interest in the determination and the analysis of the correcting terms $T_{b l}^{1, m}$ of the homogenized model.

In practice, without morphological analysis of the medium at microscopic scale, the "true" value of the parameter $a_{/ /}^{*}$ is obviously unknown, and the correcting terms $T_{b l}^{1, m}$ too, so the bias cannot be evaluated following the numerical process illustrated above. Moreover noisy measurements have to be taken into account. A specific inverse analysis has to be developed.

\section{7.-Conclusions}

A space-time homogenization approach of a transient heat conduction problem in a periodic composite material has been developed according to a homogenization approach based on an asymptotic expansion method. These mathematical results generalize the steady state analysis already developed by the authors. It leads to solve two kinds of problems, i.e. at the macroscopic and the microscopic scales. These results are available for any 3-D periodic heterogeneous structure, when the thermal contrast remains relatively low. 
Like in steady state, spatial correcting terms near the boundaries are required in the homogenized solution and evolve with time. Moreover, "short time" correcting terms are also needed (but in the whole spatial domain) to fit the heterogeneous solution with a good accuracy.

Following this approach, it was shown how the effective thermal diffusivity of such material may be biased, when it is estimated by fitting the experimental temperature rise from a laser flash experiment with the homogenized temperature computed without correcting terms. Numerical examples illustrate this bias, and suggest why a specific inverse analysis has to be developed.

\section{References}

[1] M Thomas (2008)- Thermal properties of composite materials- Experimental characterization and microstructural approach; PhD Thesis (english version), Université de Nantes.

[2] M Bornet, T Bretheau, P Gilormini (2001). Homogénéisation en mécanique des matériaux 1- Hermes Science- Paris.

[3] A Degiovanni , M Laurent (1986)- New Thermal-Diffusivity Identification applied to Flash Method- Revue de Physique Appliquée, 21 (3), pp 229-237.

[4]- W J Parker, R J Jenkins, C P Butler, G Abott (1961) - Flash method for determining thermal diffusivity, heat capacity and thermal conductivity- J. Appl. Phys. 32 (9), 1679-1684.

[5] A Anderson (1976)- Thermal conductivity of some rubbers under pressure by the transient hot-wire method J. Appl. Phys. 47, 2424-2426

[6] M Thomas, N Boyard, N Lefèvre, Y Jarny, D Delaunay (2010) - An experimental device for the simultaneous estimation of the thermal conductivity 3-D tensor and the specific heat of orthotropic composite materials Compos.Sci.Technol. 53, 5487-5498.

[7] M Thomas, N Boyard, L Perez, Y Jarny, D Delaunay (2008) - Representative volume element of anisotropic unidirectional carbon-epoxy composite with high fiber volume fraction - Compos. Sci. Technol., 68, 3184-3192.

[8] A Bensoussan, JL Lions, G Papanicolaou (1978)- Asymptotic Analysis for periodic structures - North Holland Ed., Amsterdam.

[9] Homogenization techniques for composites media -E Sanchez-Palenzia, A Zaoui editors, (1987) Lecture Notes in Physics, Volume 272 ,Springer Berlin Heidelberg.

[10] J Auriault, C Boutin, C Geindreau (2010) - Homogenization of Coupled Phenomena in Heterogeneous Media - J Wiley \& Sons.

[11] O Fudym, JC Batsale, D Lecomte (2004) - Heat diffusion at the boundary of stratified media Homogenized temperature field and thermal constriction - Int. J. of Heat and Mass Transfer 47, 2437-2447.

[12] N Buannic, P Cartraud (2001) - Higher-order effective modeling of periodic heterogeneous beams - Part 2 : derivation of the proper boundary conditions for the interior asymptotic solution - Int. J. of Solids and Structures, 38, 7163-7180.

[13] A Matine, N Boyard, P Cartraud, G Legrain, Y Jarny (2013) - Modeling of thermo-physical properties in heterogeneous periodic media according to a multi-scale approach: effective conductivity tensor and edge effects - Int. J. of Heat and Mass Transfer, 62, 586-603.

[14] H Dumontet (1986)-Study of boundary layer problem in elastic composite materials- RAIRO-Modélisation mathématique et analyse numérique, tome $20, \mathrm{n}^{\circ} 2,265-286$.

[15] J V Beck, B Blackwell C St. Clair. (1985) - Inverse Heat Conduction, Ill-posed problems. WileyInterscience, New York.

[16] M Kaminski (2003) - Homogenization of transient heat transfer problems for some composite materials Int. J. of Eng. Sci. 41, 1-29.

[17] F Larsson, K Runesson, F Su (2010) - Variationally consistent computational homogenization of transient heat flow- Int. J. Numer. Methods Eng., 81 (13), 1659-1686.

[18] A Dasgupta, R K Agarwal (1992) - Orthotropic thermal conductivity of plain-weave fabric composites using a homogenization technique - J. of Comp. Mat. 26 (18), 2736-2759. 
[19] M Amar, M Tarallo, S Terracini (1999) - On the exponential decay for boundary layer problems - C.R. Acad. Sci., Paris, 328, serie 1, 1139-1144.

[20] H S Carslaw, J C Jaeger (1959), Conduction of Heat in Solids, Oxford at the Clarendon Press.

[21] A Piatnitski (1980) - A parabolic equation with rapidly oscillating coefficients. Mosc. Univ. Math. Bull, 35 (3), 35-42.

[22] D Aubry, G Puel (2010) - Ccf modelling with use of a two-timescale homogenization model. Procedia Engineering 2 (1), 787- 796.

[23] Q Yu, J Fish (2002) - Multiscale asymptotic homogenization for multiphysics problems with multiple spatial and temporal scales : a coupled thermo-viscoelastic example problem. Int. J. of Solids and Structures, 39 (26), 6429-6452.

[24] J Fish, W Chen (2004) - Space-time multiscale model for wave propagation in heterogeneous media. Comput. Methods Appl. Mech. Engrg., 193 (45-47),4837-4856. 


\section{TABLE HEADINGS}

Table 1: Thermal and geometrical data for the multilayered periodic structure- Cases \#1 to \#3 Table 2: Thermal and geometrical data for the multilayered periodic structure- Case \#4 Table 3: Biased estimations of the thermal diffusivity component $a_{/ /}$- Influence of the sensor location

\section{FIGURE CAPTIONS}

Figure 1: Spatial domain $\Omega$ of the heterogeneous periodic medium and the associated periodic cell $Y$

Figure 2: Schematic description of the multi-layered medium and the periodic cell- Insulating layer (grey), conductive layer (blank). Case \#1: non isothermal initial condition and periodic boundary conditions.

Figure 3: Numerical solutions $\omega_{i}(\boldsymbol{y}), \mathrm{i}=1,2$ computed on the periodic cell $\mathrm{Y}$

Figure 4: Comparison of the homogenized and heterogeneous temperatures- Case \#1 $4 a:\left(x_{1}=5 \mathrm{~mm} ; x_{2}=5.3 \mathrm{~mm}\right)$ - insulating layer $/ 4 \mathrm{~b}:$ at $\left(x_{1}=5 \mathrm{~mm} ; x_{2}=4.8 \mathrm{~mm}\right)$ - in the conductive layer

Figure 5: Comparison of the corrected homogenized and heterogeneous temperatures for different locationsCase \#2 / 5a-b: $\left(\mathrm{x}_{1}=5 \mathrm{~mm} ; \mathrm{x}_{2}=5.3 \mathrm{~mm}\right)-; 5 \mathrm{c}-\mathrm{d}:\left(\mathrm{x}_{1}=5 \mathrm{~mm} ; \mathrm{x}_{2}=2.8 \mathrm{~mm}\right)-; 5 \mathrm{e}-\mathrm{f}:\left(\mathrm{x}_{1}=5 \mathrm{~mm} ; \mathrm{x}_{2}=\right.$ $1.3 \mathrm{~mm})$

Figure 6: Presentation of the sub-domains $G_{m}$ into the spatial domain $\Omega$, in the normal direction $\left(e_{1}\right)$ to the boundaries $\Gamma_{\mathrm{m}}$

Figure 7: Presentation of the multi-layered medium and the periodic cell - Case \#3: isothermal initial condition together with a Neumann and a Dirichlet boundary conditions

Figure 8: (8a)-(8c) Heterogeneous and corrected homogenized temperatures on the front face $\left(\mathrm{x}_{1}=0\right)$ (8b)-(8d) Spatial variations of the correcting terms close to the front face -Case \#3.

(8a)-(8b) $\left.x_{2}=5.3 \mathrm{~mm}\right)$, insulating layer, (8c)-(8d) $\left.x_{2}=5.8 \mathrm{~mm}\right)$, conductive layer

Figure 9: Multi-layered medium -Case \#4: heat pulse on the front face- isothermal initial condition together with Fourier boundary conditions are applied.

Figure 10: Heterogeneous temperatures $T^{\varepsilon}(x, t)$ computed for a laser flash experiment - Case \#4. (10.a): inside the slab, at short times : $\mathrm{t}=0.001,0.01,0.05,0.1 \mathrm{~s}$. (10.b) on the back face, at different locations.

Figure 11: Homogenized temperatures on the back face, computed with and without the correcting terms, compared to the heterogeneous solution- Case \#4. (11a): insulating layer $\left(x_{2}=5.3 \mathrm{~mm}\right),(11 \mathrm{~b})$ conductive layer at $\left(x_{2}=5.8 \mathrm{~mm}\right)$.

Figure 12: Homogenized heat flux density on the back face, computed without and with the correcting terms compared to the heterogeneous solution- Case \#4. (12a) at $\mathrm{x}_{2}=5.3 \mathrm{~mm}$, insulating layer, (12b) at $\mathrm{x}_{2}=5.8 \mathrm{~mm}$ conductive layer, (12c) heat flux density component in the direction $\mathrm{e}_{1}$, at $\mathrm{t}=0.5 \mathrm{~s},(12 \mathrm{~d})$ heat flux density component in the direction $\mathrm{e}_{2}$, at $\mathrm{t}=0.5 \mathrm{~s}$.

Figure 13: Temperature residual errors resulting of the data matching process on the back face - Case \#4 / Influence of the sensor location. (a) insulating layer $\left(\mathrm{x}_{2}=5.3 \mathrm{~mm}\right)$, (b) conductive layer $\left(\mathrm{x}_{2}=5.8 \mathrm{~mm}\right)$, (c) average response.

Figure A.1: Back face temperatures in a homogeneous slab, resulting of a heat pulse on the front faceInfluence of the heat losses. 


\section{Appendix 1: The analytical response of a LF experiment for a 1-D homogeneous}

\section{medium}

The temperature rise $T_{\text {exact-1D }}(x=e, t)$ on the back face of a homogeneous slab, resulting of a heat pulse on the front face, can be obtained analytically by solving the 1-D heat conduction equation [20]. The initial temperature field is uniform and equal to the external temperature, which is assumed to be constant during the experiment. Fourier conditions are considered on both faces. The following data are assumed to be given:

- the thickness $e$ of the slab.

- the energy density $\varphi$ of the heat pulse.

- the heat transfer coefficient $h$

- the heat capacity $\left(\rho C_{p}\right)^{*}$ and the heat conductivity $k_{11}^{*}$

- $\quad$ and/or the thermal diffusivity $a_{/ /}=k_{11}^{*} /\left(\rho C_{p}\right)^{*}$.

$$
\begin{gathered}
T_{\text {exact-1D }}(x=e, t)=\frac{2 \varphi}{\left(\rho C_{p}\right)^{*}} \sum_{n} \frac{\Psi\left(\alpha_{n}, B i\right)}{N\left(\alpha_{n}, B i\right)} \exp \left(-a_{/ /} t\left(\frac{\alpha_{n}}{e}\right)^{2}\right) \\
\text { where } B i=\frac{h e}{k_{11}^{*}} ; N\left(\alpha_{n}, B i\right)=\alpha_{n}+\frac{B i}{\alpha_{n}}(B i+2) ; \\
\Psi\left(\alpha_{n}, B i\right)=\alpha_{n} \cos \left(\alpha_{n}\right)+B i \cdot \sin \left(\alpha_{n}\right) \\
\text { and } \alpha_{n,} n=1, . . \text { are the solutions of } \tan (\alpha)=\frac{2 . B i \cdot \alpha}{\alpha^{2}-B i^{2}}
\end{gathered}
$$

The solutions plotted below, are computed on the back face with $e=5 \mathrm{~mm}, \varphi=2 * 10^{2} \mathrm{~J} \mathrm{~m}^{-2}$ $k_{11}^{*}=0.550\left(\mathrm{~W} \mathrm{~m}^{-1} \mathrm{~K}^{-1}\right), \quad a_{/ /}=18.3 * 10^{-6} \mathrm{~m}^{2} \mathrm{~s}^{-1}$ and for three distinct values of the heat transfer coefficient $h=10,50,100 \mathrm{~W} \mathrm{~m}^{-2} \mathrm{~K}^{-1}$, in order to illustrate the influence of the heat losses.

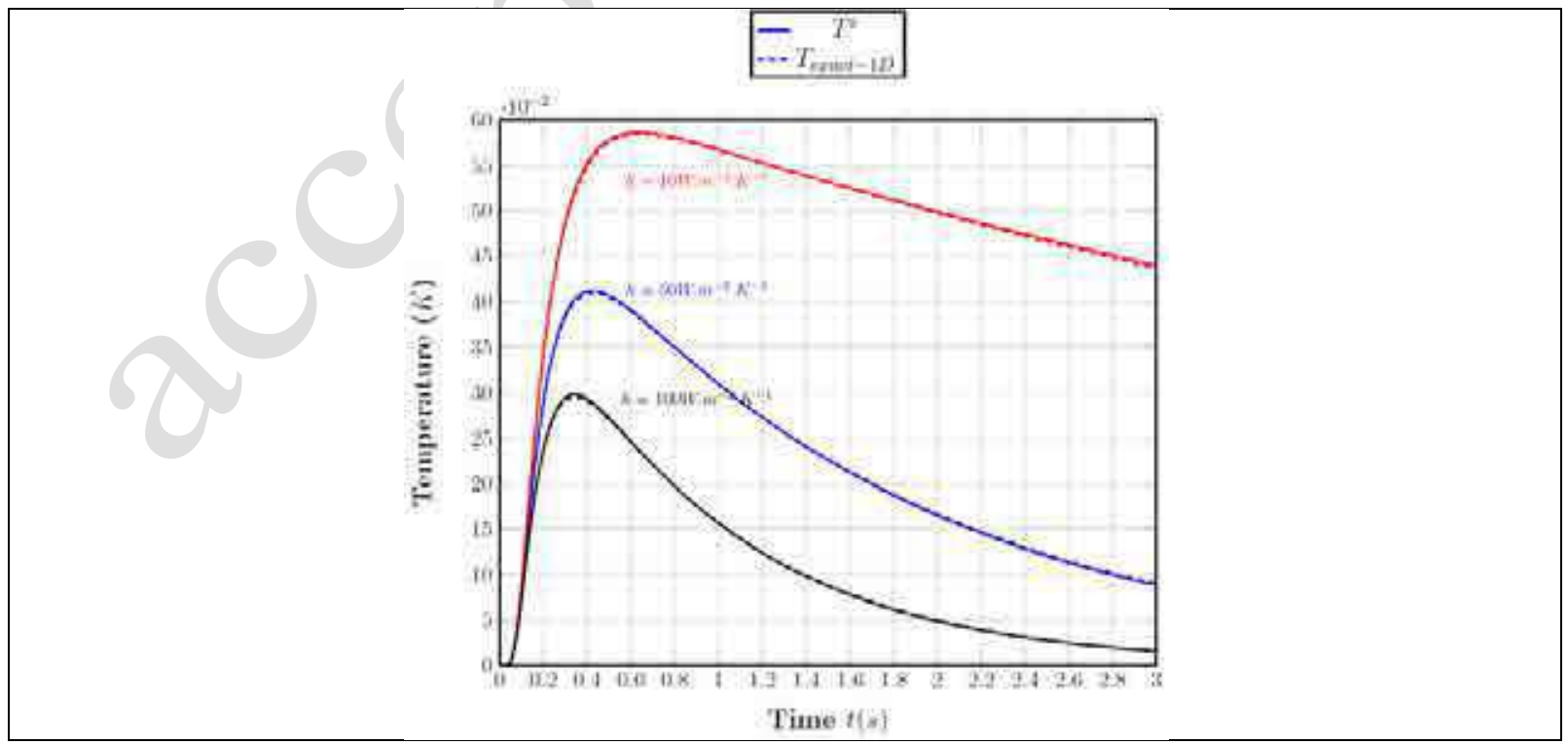

Figure A.1: Back face temperatures in a homogeneous slab, resulting of a heat pulse on the front faceInfluence of the heat losses 